\title{
Barnes-type Narumi of the second kind and Barnes-type Peters of the second kind hybrid polynomials
}

\section{Dae San Kim ${ }^{1}$, Taekyun Kim²*, Takao Komatsu ${ }^{3}$ and Hyuck In Kwon ${ }^{2}$}

${ }^{*}$ Correspondence: tkkim@kw.ac.kr ${ }^{2}$ Department of Mathematics, Kwangwoon University, Seoul,

139-701, Republic of Korea Full list of author information is available at the end of the article

\begin{abstract}
In this paper, by considering Barnes-type Narumi polynomials of the second kind and Barnes-type Peters polynomials of the second kind, we define and investigate the hybrid polynomials of these polynomials. From the properties of Sheffer sequences of these polynomials arising from umbral calculus, we derive new and interesting identities.

MSC: $05 \mathrm{~A} 15 ; 05 \mathrm{~A} 40 ; 11 \mathrm{~B} 68 ; 11 \mathrm{~B} 75 ; 11 \mathrm{~B} 83$
\end{abstract}

\section{Introduction}

In this paper, we consider the polynomials

$$
\widehat{\mathrm{NS}}_{n}(x)=\widehat{\mathrm{NS}}_{n}(x \mid a ; \lambda ; \mu)=\widehat{\mathrm{NS}}_{n}\left(x \mid a_{1}, \ldots, a_{r} ; \lambda_{1}, \ldots, \lambda_{s} ; \mu_{1}, \ldots, \mu_{s}\right)
$$

called Barnes-type Narumi of the second kind and Barnes-type Peters of the second kind hybrid polynomials, whose generating function is given by

$$
\begin{gathered}
\prod_{i=1}^{r}\left(\frac{(1+t)^{a_{i}}-1}{(1+t)^{a_{i}} \ln (1+t)}\right) \prod_{j=1}^{s}\left(\frac{(1+t)^{\lambda_{j}}}{1+(1+t)^{\lambda_{j}}}\right)^{\mu_{j}}(1+t)^{x} \\
\quad=\sum_{n=0}^{\infty} \widehat{\mathrm{NS}}_{n}\left(x \mid a_{1}, \ldots, a_{r} ; \lambda_{1}, \ldots, \lambda_{s} ; \mu_{1}, \ldots, \mu_{s}\right) \frac{t^{n}}{n !},
\end{gathered}
$$

where $a_{1}, \ldots, a_{r}, \lambda_{1}, \ldots, \lambda_{s}, \mu_{1}, \ldots, \mu_{s} \in \mathbb{C}$ with $a_{1}, \ldots, a_{r}, \lambda_{1}, \ldots, \lambda_{s} \neq 0$. When $x=0, \widehat{\mathrm{NS}}_{n}=$ $\widehat{\mathrm{NS}}_{n}(0)=\widehat{\mathrm{NS}}_{n}(0 \mid a ; \lambda ; \mu)=\widehat{\mathrm{NS}}_{n}\left(0 \mid a_{1}, \ldots, a_{r} ; \lambda_{1}, \ldots, \lambda_{s} ; \mu_{1}, \ldots, \mu_{s}\right)$ are called Barnes-type Narumi of the second kind and Barnes-type Peters of the second kind hybrid numbers.

Recall that the Barnes-type Narumi polynomials of the second kind, denoted by $\widehat{\mathrm{N}}_{n}\left(x \mid a_{1}, \ldots, a_{r}\right)$, are given by the generating function as

$$
\prod_{i=1}^{r}\left(\frac{(1+t)^{a_{i}}-1}{(1+t)^{a_{i}} \ln (1+t)}\right)(1+t)^{x}=\sum_{n=0}^{\infty} \widehat{\mathrm{N}}_{n}\left(x \mid a_{1}, \ldots, a_{r}\right) \frac{t^{n}}{n !}
$$

If $x=0$, we write $\widehat{\mathrm{N}}_{n}\left(a_{1}, \ldots, a_{r}\right)=\widehat{\mathrm{N}}_{n}\left(0 \mid a_{1}, \ldots, a_{r}\right)$. Narumi polynomials were mentioned in [1, p.127]. In addition, the Barnes-type Peters polynomials of the second kind, denoted by

\section{Springer}

@2014 Kim et al.; licensee Springer. This is an Open Access article distributed under the terms of the Creative Commons Attribution License (http://creativecommons.org/licenses/by/2.0), which permits unrestricted use, distribution, and reproduction in any medium, provided the original work is properly cited. 
$\hat{s}_{n}\left(x \mid \lambda_{1}, \ldots, \lambda_{s} ; \mu_{1}, \ldots, \mu_{s}\right)$, are given by the generating function as

$$
\prod_{j=1}^{s}\left(\frac{(1+t)^{\lambda_{j}}}{1+(1+t)^{\lambda_{j}}}\right)^{\mu_{j}}(1+t)^{x}=\sum_{n=0}^{\infty} \hat{s}_{n}\left(x \mid \lambda_{1}, \ldots, \lambda_{s} ; \mu_{1}, \ldots, \mu_{s}\right) \frac{t^{n}}{n !} .
$$

If $s=1$, then $\hat{s}_{n}(x \mid \lambda ; \mu)$ are the Peters polynomials of the second kind. Peters polynomials were mentioned in [1, p.128] and have been investigated in e.g. [2].

In this paper, by considering Barnes-type Narumi polynomials of the second kind and Barnes-type Peters polynomials of the second kind, we define and investigate the hybrid polynomials of these polynomials. From the properties of Sheffer sequences of these polynomials arising from umbral calculus, we derive new and interesting identities.

\section{Umbral calculus}

Let $\mathbb{C}$ be the complex number field and let $\mathcal{F}$ be the set of all formal power series in the variable $t$ :

$$
\mathcal{F}=\left\{f(t)=\sum_{k=0}^{\infty} \frac{a_{k}}{k !} t^{k} \mid a_{k} \in \mathbb{C}\right\} .
$$

Let $\mathbb{P}=\mathbb{C}[x]$ and let $\mathbb{P}^{*}$ be the vector space of all linear functionals on $\mathbb{P} .\langle L \mid p(x)\rangle$ is the action of the linear functional $L$ on the polynomial $p(x)$, and we recall that the vector space operations on $\mathbb{P}^{*}$ are defined by $\langle L+M \mid p(x)\rangle=\langle L \mid p(x)\rangle+\langle M \mid p(x)\rangle,\langle c L \mid p(x)\rangle=c\langle L \mid p(x)\rangle$, where $c$ is a complex constant in $\mathbb{C}$. For $f(t) \in \mathcal{F}$, let us define the linear functional on $\mathbb{P}$ by setting

$$
\left\langle f(t) \mid x^{n}\right\rangle=a_{n} \quad(n \geq 0) .
$$

In particular,

$$
\left\langle t^{k} \mid x^{n}\right\rangle=n ! \delta_{n, k} \quad(n, k \geq 0)
$$

where $\delta_{n, k}$ is Kronecker's symbol.

For $f_{L}(t)=\sum_{k=0}^{\infty} \frac{\left\langle L \mid x^{k}\right\rangle}{k !} t^{k}$, we have $\left\langle f_{L}(t) \mid x^{n}\right\rangle=\left\langle L \mid x^{n}\right\rangle$. That is, $L=f_{L}(t)$. The map $L \mapsto f_{L}(t)$ is a vector space isomorphism from $\mathbb{P}^{*}$ onto $\mathcal{F}$. Henceforth, $\mathcal{F}$ denotes both the algebra of formal power series in $t$ and the vector space of all linear functionals on $\mathbb{P}$, and so an element $f(t)$ of $\mathcal{F}$ will be thought of as both a formal power series and a linear functional. We call $\mathcal{F}$ the umbral algebra and the umbral calculus is the study of umbral algebra. The order $O(f(t))$ of a power series $f(t)(\neq 0)$ is the smallest integer $k$ for which the coefficient of $t^{k}$ does not vanish. If $O(f(t))=1$, then $f(t)$ is called a delta series; if $O(f(t))=0$, then $f(t)$ is called an invertible series. For $f(t), g(t) \in \mathcal{F}$ with $O(f(t))=1$ and $O(g(t))=0$, there exists a unique sequence $s_{n}(x)\left(\operatorname{deg} s_{n}(x)=n\right)$ such that $\left\langle g(t) f(t)^{k} \mid s_{n}(x)\right\rangle=n ! \delta_{n, k}$, for $n, k \geq 0$. Such a sequence $s_{n}(x)$ is called the Sheffer sequence for $(g(t), f(t))$ which is denoted by $s_{n}(x) \sim(g(t), f(t))$.

For $f(t), g(t) \in \mathcal{F}$, and $p(x) \in \mathbb{P}$, we have

$$
\langle f(t) g(t) \mid p(x)\rangle=\langle f(t) \mid g(t) p(x)\rangle=\langle g(t) \mid f(t) p(x)\rangle
$$


and

$$
\begin{aligned}
& f(t)=\sum_{k=0}^{\infty}\left\langle f(t) \mid x^{k}\right\rangle \frac{t^{k}}{k !}, \\
& p(x)=\sum_{k=0}^{\infty}\left\langle t^{k} \mid p(x)\right\rangle \frac{x^{k}}{k !}
\end{aligned}
$$

[1, Theorem 2.2.5]. Thus, by (6), we get

$$
t^{k} p(x)=p^{(k)}(x)=\frac{d^{k} p(x)}{d x^{k}} \quad \text { and } \quad e^{y t} p(x)=p(x+y)
$$

Sheffer sequences are characterized by the generating function of [1, Theorem 2.3.4].

Lemma 1 The sequence $s_{n}(x)$ is Sheffer for $(g(t), f(t))$ if and only if

$$
\frac{1}{g(\bar{f}(t))} e^{y \bar{f}(t)}=\sum_{k=0}^{\infty} \frac{s_{k}(y)}{k !} t^{k} \quad(y \in \mathbb{C}),
$$

where $\bar{f}(t)$ is the compositional inverse of $f(t)$.

For $s_{n}(x) \sim(g(t), f(t))$, we have the following equations [1, Theorem 2.3.7, Theorem 2.3.5, Theorem 2.3.9]:

$$
\begin{aligned}
& f(t) s_{n}(x)=n s_{n-1}(x) \quad(n \geq 0), \\
& s_{n}(x)=\sum_{j=0}^{n} \frac{1}{j !}\left\langle g(\bar{f}(t))^{-1} \bar{f}(t)^{j} \mid x^{n}\right\rangle x^{j}, \\
& s_{n}(x+y)=\sum_{j=0}^{n}\left(\begin{array}{l}
n \\
j
\end{array}\right) s_{j}(x) p_{n-j}(y),
\end{aligned}
$$

where $p_{n}(x)=g(t) s_{n}(x)$.

Assume that $p_{n}(x) \sim(1, f(t))$ and $q_{n}(x) \sim(1, g(t))$. Then the transfer formula [1, Corollary 3.8.2] is given by

$$
q_{n}(x)=x\left(\frac{f(t)}{g(t)}\right)^{n} x^{-1} p_{n}(x) \quad(n \geq 1) .
$$

For $s_{n}(x) \sim(g(t), f(t))$, and $r_{n}(x) \sim(h(t), l(t))$, assume that

$$
s_{n}(x)=\sum_{m=0}^{n} C_{n, m} r_{m}(x) \quad(n \geq 0) .
$$

Then we have $[1, \mathrm{p} .132]$

$$
C_{n, m}=\frac{1}{m !}\left\langle\frac{h(\bar{f}(t))}{g(\bar{f}(t))} l(\bar{f}(t))^{m} \mid x^{n}\right\rangle .
$$




\section{Main results}

For convenience, we introduce an Appell sequence of polynomials $F_{n}\left(a_{1}, \ldots, a_{r}\right)\left(a_{1}, \ldots\right.$, $\left.a_{r} \neq 0\right)$, defined by

$$
\prod_{i=1}^{r}\left(\frac{e^{a_{i} t}-1}{t}\right) e^{x t}=\sum_{n=0}^{\infty} F_{n}\left(x \mid a_{1}, \ldots, a_{r}\right) \frac{t^{n}}{n !} .
$$

If $x=0$, we write $F_{n}\left(a_{1}, \ldots, a_{r}\right)=F_{n}\left(0 \mid a_{1}, \ldots, a_{r}\right)$. By [1, Theorem 2.5.8] with $y=0$, we have

$$
F_{n}\left(x \mid a_{1}, \ldots, a_{r}\right)=\sum_{m=0}^{n}\left(\begin{array}{c}
n \\
m
\end{array}\right) F_{n-m}\left(a_{1}, \ldots, a_{r}\right) x^{m}
$$

More precisely, one can show that

$$
F_{n}\left(x \mid a_{1}, \ldots, a_{r}\right)=\sum_{i=0}^{n} \sum_{l_{1}+\cdots+l_{r}=i} \frac{i !}{(i+r) !}\left(\begin{array}{c}
n \\
i
\end{array}\right)\left(\begin{array}{c}
i+r \\
l_{1}+1, \ldots, l_{r}+1
\end{array}\right) a_{1}^{l_{1}+1} \cdots a_{r}^{l_{r}+1} x^{n-i},
$$

where

$$
\left(\begin{array}{c}
i+r \\
l_{1}+1, \ldots, l_{r}+1
\end{array}\right)=\frac{(i+r) !}{\left(l_{1}+1\right) ! \cdots\left(l_{r}+1\right) !} .
$$

Remember that the generalized Barnes-type Euler polynomials $E_{n}\left(x \mid \lambda_{1}, \ldots, \lambda_{s} ; \mu_{1}, \ldots, \mu_{s}\right)$ are defined by the generating function

$$
\prod_{j=1}^{s}\left(\frac{2}{1+e^{\lambda_{j} t}}\right)^{\mu_{j}} e^{x t}=\sum_{n=0}^{\infty} E_{n}\left(x \mid \lambda_{1}, \ldots, \lambda_{s} ; \mu_{1}, \ldots, \mu_{s}\right) \frac{t^{n}}{n !} .
$$

If $\mu_{1}=\cdots=\mu_{s}=1$, then $E_{n}\left(x \mid \lambda_{1}, \ldots, \lambda_{s}\right)=E_{n}\left(x \mid \lambda_{1}, \ldots, \lambda_{s} ; 1, \ldots, 1\right)$ are called the Barnestype Euler polynomials. If further $\lambda_{1}=\cdots=\lambda_{s}=1$, then $E_{n}^{(r)}(x)=E_{n}(x \mid 1, \ldots, 1 ; 1, \ldots, 1)$ are called the Euler polynomials of order $s$. If $x=0$, we write $E_{n}\left(\lambda_{1}, \ldots, \lambda_{s} ; \mu_{1}, \ldots, \mu_{s}\right)=$ $E_{n}\left(0 \mid \lambda_{1}, \ldots, \lambda_{s} ; \mu_{1}, \ldots, \mu_{s}\right)$. By [1, Theorem 2.5.8] with $y=0$, we have

$$
E_{n}\left(x \mid \lambda_{1}, \ldots, \lambda_{s} ; \mu_{1}, \ldots, \mu_{s}\right)=\sum_{m=0}^{n}\left(\begin{array}{c}
n \\
m
\end{array}\right) E_{n-m}\left(\lambda_{1}, \ldots, \lambda_{s} ; \mu_{1}, \ldots, \mu_{s}\right) x^{m}
$$

We note that

$$
\begin{array}{r}
t F_{n}\left(x \mid a_{1}, \ldots, a_{r}\right)=\frac{d}{d x} F_{n}\left(x \mid a_{1}, \ldots, a_{r}\right)=n F_{n-1}\left(x \mid a_{1}, \ldots, a_{r}\right), \\
t E_{n}\left(x \mid \lambda_{1}, \ldots, \lambda_{s} ; \mu_{1}, \ldots, \mu_{s}\right)=\frac{d}{d x} E_{n}\left(x \mid \lambda_{1}, \ldots, \lambda_{s} ; \mu_{1}, \ldots, \mu_{s}\right) \\
=n E_{n-1}\left(x \mid \lambda_{1}, \ldots, \lambda_{s} ; \mu_{1}, \ldots, \mu_{s}\right) .
\end{array}
$$

From the definition (1), $\widehat{\mathrm{NS}}_{n}\left(x \mid a_{1}, \ldots, a_{r} ; \lambda_{1}, \ldots, \lambda_{s} ; \mu_{1}, \ldots, \mu_{s}\right)$ is the Sheffer sequence for the pair

$$
g(t)=\prod_{i=1}^{r}\left(\frac{t e^{a_{i} t}}{e^{a_{i} t}-1}\right) \prod_{j=1}^{s}\left(\frac{1+e^{\lambda_{j} t}}{e^{\lambda_{j} t}}\right)^{\mu_{j}} \quad \text { and } \quad f(t)=e^{t}-1 .
$$


So,

$$
\widehat{\mathrm{NS}}_{n}\left(x \mid a_{1}, \ldots, a_{r} ; \lambda_{1}, \ldots, \lambda_{s} ; \mu_{1}, \ldots, \mu_{s}\right) \sim\left(\prod_{i=1}^{r}\left(\frac{t e^{a_{i} t}}{e^{a_{i} t}-1}\right) \prod_{j=1}^{s}\left(\frac{1+e^{\lambda_{j} t}}{e^{\lambda_{j} t}}\right)^{\mu_{j}}, e^{t}-1\right) .
$$

\subsection{Explicit expressions}

Let $(n)_{j}=n(n-1) \cdots(n-j+1)(j \geq 1)$ with $(n)_{0}=1$. The (signed) Stirling numbers of the first kind $S_{1}(n, m)$ are defined by

$$
(x)_{n}=\sum_{m=0}^{n} S_{1}(n, m) x^{m}
$$

\section{Theorem 1}

$$
\begin{aligned}
& \widehat{\mathrm{NS}}_{n}\left(x \mid a_{1}, \ldots, a_{r} ; \lambda_{1}, \ldots, \lambda_{s} ; \mu_{1}, \ldots, \mu_{s}\right) \\
& =2^{-\sum_{j=1}^{s} \mu_{j}} \sum_{m=0}^{n} \sum_{l=0}^{m}(-1)^{m}\left(\begin{array}{c}
m \\
l
\end{array}\right) S_{1}(n, m) E_{m-l}\left(\lambda_{1}, \ldots, \lambda_{s} ; \mu_{1}, \ldots, \mu_{s}\right) \\
& \times F_{l}\left(-x \mid a_{1}, \ldots, a_{r}\right) \\
& =2^{-\sum_{j=1}^{s} \mu_{j}} \sum_{m=0}^{n} \sum_{l=0}^{m}\left(\begin{array}{c}
m \\
l
\end{array}\right) S_{1}(n, m) E_{m-l}\left(\lambda_{1}, \ldots, \lambda_{s} ; \mu_{1}, \ldots, \mu_{s}\right) \\
& \times F_{l}\left(x+\sum_{j=1}^{s} \lambda_{j} \mu_{j}-\sum_{i=1}^{r} a_{i} \mid a_{1}, \ldots, a_{r}\right) \\
& =2^{-\sum_{j=1}^{s} \mu_{j}} \sum_{m=0}^{n} \sum_{l=0}^{m}(-1)^{m}\left(\begin{array}{c}
m \\
l
\end{array}\right) S_{1}(n, m) F_{m-l}\left(a_{1}, \ldots, a_{r}\right) \\
& \times E_{l}\left(-x \mid \lambda_{1}, \ldots, \lambda_{s} ; \mu_{1}, \ldots, \mu_{s}\right) \\
& =\sum_{j=0}^{n} \sum_{l=j}^{n}\left(\begin{array}{l}
n \\
l
\end{array}\right) S_{1}(l, j) \widehat{\mathrm{NS}}_{n-l} x^{j} \\
& =\sum_{l=0}^{n}\left(\begin{array}{l}
n \\
l
\end{array}\right) \hat{s}_{n-l}\left(\lambda_{1}, \ldots, \lambda_{r} ; \mu_{1}, \ldots, \mu_{r}\right) \widehat{N}_{l}\left(x \mid a_{1}, \ldots, a_{r}\right), \\
& =\sum_{l=0}^{n}\left(\begin{array}{l}
n \\
l
\end{array}\right) \widehat{N}_{n-l}\left(a_{1}, \ldots, a_{r}\right) \hat{s}_{l}\left(x \mid \lambda_{1}, \ldots, \lambda_{s} ; \mu_{1}, \ldots, \mu_{s}\right) .
\end{aligned}
$$

Proof Since

$$
\prod_{i=1}^{r}\left(\frac{t e^{a_{i} t}}{e^{a_{i} t}-1}\right) \prod_{j=1}^{s}\left(\frac{1+e^{\lambda_{j} t}}{e^{\lambda_{j} t}}\right)^{\mu_{j}} \widehat{\mathrm{NS}}_{n}\left(x \mid a_{1}, \ldots, a_{r} ; \lambda_{1}, \ldots, \lambda_{s} ; \mu_{1}, \ldots, \mu_{s}\right) \sim\left(1, e^{t}-1\right)
$$

and

$$
(x)_{n} \sim\left(1, e^{t}-1\right)
$$


Kim et al. Journal of Inequalities and Applications 2014, 2014:376

Page 6 of 23

http://www.journalofinequalitiesandapplications.com/content/2014/1/376

we have

$$
\begin{aligned}
& \widehat{\mathrm{NS}}_{n}\left(x \mid a_{1}, \ldots, a_{r} ; \lambda_{1}, \ldots, \lambda_{s} ; \mu_{1}, \ldots, \mu_{s}\right) \\
& =\prod_{i=1}^{r}\left(\frac{e^{a_{i} t}-1}{t e^{a_{i} t}}\right) \prod_{j=1}^{s}\left(\frac{e^{\lambda_{j} t}}{1+e^{\lambda_{j} t}}\right)^{\mu_{j}}(x)_{n} \\
& =\sum_{m=0}^{n} S_{1}(n, m) \prod_{i=1}^{r}\left(\frac{e^{a_{i} t}-1}{t e^{a_{i} t}}\right) \prod_{j=1}^{s}\left(\frac{e^{\lambda_{j} t}}{1+e^{\lambda_{j} t}}\right)^{\mu_{j}} x^{m} \\
& =2^{-\sum_{j=1}^{s} \mu_{j}} \sum_{m=0}^{n} S_{1}(n, m) \prod_{i=1}^{r}\left(\frac{e^{-a_{i} t}-1}{-t}\right) \prod_{j=1}^{s}\left(\frac{2}{1+e^{-\lambda_{j} t}}\right)^{\mu_{j}} x^{m} \\
& =2^{-\sum_{j=1}^{s} \mu_{j}} \sum_{m=0}^{n} S_{1}(n, m) \prod_{i=1}^{r}\left(\frac{e^{-a_{i} t}-1}{-t}\right)(-1)^{m} E_{m}\left(-x \mid \lambda_{1}, \ldots, \lambda_{s} ; \mu_{1}, \ldots, \mu_{s}\right) \\
& =2^{-\sum_{j=1}^{s} \mu_{j}} \sum_{m=0}^{n} S_{1}(n, m) \prod_{i=1}^{r}\left(\frac{e^{-a_{i} t}-1}{-t}\right)(-1)^{m} \\
& \times \sum_{l=0}^{m}\left(\begin{array}{c}
m \\
l
\end{array}\right) E_{m-l}\left(\lambda_{1}, \ldots, \lambda_{s} ; \mu_{1}, \ldots, \mu_{s}\right)(-x)^{l} \\
& =2^{-\sum_{j=1}^{s} \mu_{j}} \sum_{m=0}^{n}(-1)^{m} S_{1}(n, m) \\
& \times \sum_{l=0}^{m}(-1)^{l}\left(\begin{array}{c}
m \\
l
\end{array}\right) E_{m-l}\left(\lambda_{1}, \ldots, \lambda_{s} ; \mu_{1}, \ldots, \mu_{s}\right) \prod_{i=1}^{r}\left(\frac{e^{-a_{i} t}-1}{-t}\right) x^{l} \\
& =2^{-\sum_{j=1}^{s} \mu_{j}} \sum_{m=0}^{n}(-1)^{m} S_{1}(n, m) \\
& \times \sum_{l=0}^{m}(-1)^{l}\left(\begin{array}{c}
m \\
l
\end{array}\right) E_{m-l}\left(\lambda_{1}, \ldots, \lambda_{s} ; \mu_{1}, \ldots, \mu_{s}\right)(-1)^{l} F_{l}\left(-x \mid a_{1}, \ldots, a_{r}\right) .
\end{aligned}
$$

So, we get (13).

We can obtain an alternative expression (14) for $\widehat{\mathrm{NS}}_{n}(x)$ as follows:

$$
\begin{aligned}
\widehat{\mathrm{NS}}_{n}(x)= & 2^{-\sum_{j=1}^{s} \mu_{j}} \sum_{m=0}^{n} S_{1}(n, m) e^{\left(\sum_{j=1}^{s} \lambda_{j} \mu_{j}-\sum_{i=1}^{r} a_{i}\right) t} \\
& \times \prod_{i=1}^{r}\left(\frac{e^{a_{i} t}-1}{t}\right) \prod_{j=1}^{s}\left(\frac{2}{1+e^{\lambda_{j} t}}\right)^{\mu_{j}} x^{m} \\
= & 2^{-\sum_{j=1}^{s} \mu_{j}} \sum_{m=0}^{n} S_{1}(n, m) e^{\left(\sum_{j=1}^{s} \lambda_{j} \mu_{j}-\sum_{i=1}^{r} a_{i}\right) t} \\
& \times \prod_{i=1}^{r}\left(\frac{e^{a_{i} t}-1}{t}\right) E_{m}\left(x \mid \lambda_{1}, \ldots, \lambda_{s} ; \mu_{1}, \ldots, \mu_{s}\right) \\
= & 2^{-\sum_{j=1}^{s} \mu_{j}} \sum_{m=0}^{n} S_{1}(n, m) e^{\left(\sum_{j=1}^{s} \lambda_{j} \mu_{j}-\sum_{i=1}^{r} a_{i}\right) t} \\
& \times \prod_{i=1}^{r}\left(\frac{e^{a_{i} t}-1}{t}\right) \sum_{l=0}^{m}\left(\begin{array}{c}
m \\
l
\end{array}\right) E_{m-l}\left(\lambda_{1}, \ldots, \lambda_{s} ; \mu_{1}, \ldots, \mu_{s}\right) x^{l}
\end{aligned}
$$




$$
\begin{aligned}
= & 2^{-\sum_{j=1}^{s} \mu_{j}} \sum_{m=0}^{n} \sum_{l=0}^{m}\left(\begin{array}{c}
m \\
l
\end{array}\right) S_{1}(n, m) E_{m-l}\left(\lambda_{1}, \ldots, \lambda_{s} ; \mu_{1}, \ldots, \mu_{s}\right) \\
& \times e^{\left(\sum_{j=1}^{s} \lambda_{j} \mu_{j}-\sum_{i=1}^{r} a_{i}\right) t} \prod_{i=1}^{r}\left(\frac{e^{a_{i} t}-1}{t}\right) x^{l} \\
= & 2^{-\sum_{j=1}^{s} \mu_{j}} \sum_{m=0}^{n} \sum_{l=0}^{m}\left(\begin{array}{c}
m \\
l
\end{array}\right) S_{1}(n, m) E_{m-l}\left(\lambda_{1}, \ldots, \lambda_{s} ; \mu_{1}, \ldots, \mu_{s}\right) \\
& \times F_{l}\left(x+\sum_{j=1}^{s} \lambda_{j} \mu_{j}-\sum_{i=1}^{r} a_{i} \mid a_{1}, \ldots, a_{r}\right) .
\end{aligned}
$$

From the proof of (13),

$$
\begin{aligned}
\widehat{\mathrm{NS}}_{n}\left(x \mid a_{1}, \ldots, a_{r} ; \lambda_{1}, \ldots, \lambda_{s} ; \mu_{1}, \ldots, \mu_{s}\right) \\
=2^{-\sum_{j=1}^{s} \mu_{j}} \sum_{m=0}^{n} S_{1}(n, m) \prod_{j=1}^{s}\left(\frac{2}{1+e^{-\lambda_{j} t}}\right)^{\mu_{j}} \prod_{i=1}^{r}\left(\frac{e^{-a_{i} t}-1}{-t}\right) x^{m} \\
=2^{-\sum_{j=1}^{s} \mu_{j}} \sum_{m=0}^{n} S_{1}(n, m) \prod_{j=1}^{s}\left(\frac{2}{1+e^{-\lambda_{j} t}}\right)^{\mu_{j}}(-1)^{m} F_{m}\left(-x \mid a_{1}, \ldots, a_{r}\right) \\
=2^{-\sum_{j=1}^{s} \mu_{j}} \sum_{m=0}^{n}(-1)^{m} S_{1}(n, m) \prod_{j=1}^{s}\left(\frac{2}{1+e^{-\lambda_{j} t}}\right)^{\mu_{j}} \sum_{l=0}^{m}\left(\begin{array}{c}
m \\
l
\end{array}\right) F_{m-l}\left(a_{1}, \ldots, a_{r}\right)(-x)^{l} \\
=2^{-\sum_{j=1}^{s} \mu_{j}} \sum_{m=0}^{n}(-1)^{m} S_{1}(n, m) \sum_{l=0}^{m}\left(\begin{array}{c}
m \\
l
\end{array}\right) F_{m-l}\left(a_{1}, \ldots, a_{r}\right)(-1)^{l} \prod_{j=1}^{s}\left(\frac{2}{1+e^{-\lambda_{j} t}}\right)^{\mu_{j}} x^{l} \\
=2^{-\sum_{j=1}^{s} \mu_{j}} \sum_{m=0}^{n}(-1)^{m} S_{1}(n, m) \sum_{l=0}^{m}\left(\begin{array}{c}
m \\
l
\end{array}\right) F_{m-l}\left(a_{1}, \ldots, a_{r}\right) E_{l}\left(-x \mid \lambda_{1}, \ldots, \lambda_{s} ; \mu_{1}, \ldots, \mu_{s}\right) \\
=2^{-\sum_{j=1}^{s} \mu_{j}} \sum_{m=0}^{n} \sum_{l=0}^{m}(-1)^{m}\left(\begin{array}{c}
m \\
l
\end{array}\right) S_{1}(n, m) F_{m-l}\left(a_{1}, \ldots, a_{r}\right) E_{l}\left(-x \mid \lambda_{1}, \ldots, \lambda_{s} ; \mu_{1}, \ldots, \mu_{s}\right),
\end{aligned}
$$

which is the identity (15).

By (12), we have

$$
\begin{aligned}
& \left\langle g(\bar{f}(t))^{-1} \bar{f}(t)^{j} \mid x^{n}\right\rangle \\
& \quad=\left\langle\prod_{i=1}^{r}\left(\frac{(1+t)^{a_{i}}-1}{(1+t)^{a_{i}} \ln (1+t)}\right) \prod_{j=1}^{s}\left(\frac{(1+t)^{\lambda_{j}}}{1+(1+t)^{\lambda_{j}}}\right)^{\mu_{j}}(\ln (1+t))^{j} \mid x^{n}\right\rangle \\
& \quad=\left\langle\prod_{i=1}^{r}\left(\frac{(1+t)^{a_{i}}-1}{(1+t)^{a_{i}} \ln (1+t)}\right) \prod_{j=1}^{s}\left(\frac{(1+t)^{\lambda_{j}}}{1+(1+t)^{\lambda_{j}}}\right)^{\mu_{j}} \mid j ! \sum_{l=j}^{\infty} S_{1}(l, j) \frac{t^{l}}{l !} x^{n}\right\rangle \\
& \quad=j ! \sum_{l=j}^{n}\left(\begin{array}{l}
n \\
l
\end{array}\right) S_{1}(l, j)\left\langle\prod_{i=1}^{r}\left(\frac{(1+t)^{a_{i}}-1}{(1+t)^{a_{i}} \ln (1+t)}\right) \prod_{j=1}^{s}\left(\frac{(1+t)^{\lambda_{j}}}{1+(1+t)^{\lambda_{j}}}\right)^{\mu_{j}} \mid x^{n-l}\right\rangle \\
& \quad=j ! \sum_{l=j}^{n}\left(\begin{array}{l}
n \\
l
\end{array}\right) S_{1}(l, j)\left\langle\sum_{i=0}^{\infty} \widehat{\mathrm{NS}}_{i} \frac{t^{i}}{i !} \mid x^{n-l}\right\rangle=j ! \sum_{l=j}^{n}\left(\begin{array}{l}
n \\
l
\end{array}\right) S_{1}(l, j) \widehat{\mathrm{NS}}_{n-l} .
\end{aligned}
$$

By (9), we get the identity (16). 
Next,

$$
\begin{aligned}
\widehat{\mathrm{NS}}_{n} & \left(y \mid a_{1}, \ldots, a_{r} ; \lambda_{1}, \ldots, \lambda_{s} ; \mu_{1}, \ldots, \mu_{s}\right) \\
& =\left\langle\sum_{i=0}^{\infty} \widehat{\mathrm{NS}}_{i}\left(y \mid a_{1}, \ldots, a_{r} ; \lambda_{1}, \ldots, \lambda_{s} ; \mu_{1}, \ldots, \mu_{s}\right) \frac{t^{i}}{i !} \mid x^{n}\right\rangle \\
& \left\langle\prod_{i=1}^{r}\left(\frac{(1+t)^{a_{i}}-1}{(1+t)^{a_{i}} \ln (1+t)}\right) \prod_{j=1}^{s}\left(\frac{(1+t)^{\lambda_{j}}}{1+(1+t)^{\lambda_{j}}}\right)^{\mu_{j}}(1+t)^{y} \mid x^{n}\right\rangle \\
& \left\langle\prod_{j=1}^{s}\left(\frac{(1+t)^{\lambda_{j}}}{1+(1+t)^{\lambda_{j}}}\right)^{\mu_{j}} \mid \prod_{i=1}^{r}\left(\frac{(1+t)^{a_{i}}-1}{(1+t)^{a_{i}} \ln (1+t)}\right)(1+t)^{y} x^{n}\right\rangle \\
& =\left\langle\prod_{j=1}^{s}\left(\frac{(1+t)^{\lambda_{j}}}{1+(1+t)^{\lambda_{j}}}\right)^{\mu_{j}} \mid \sum_{l=0}^{\infty} \widehat{N}_{l}\left(y \mid a_{1}, \ldots, a_{r}\right) \frac{t^{l}}{l !} x^{n}\right\rangle \\
& =\sum_{l=0}^{n}\left(\begin{array}{c}
n \\
l
\end{array}\right) \widehat{N}_{l}\left(y \mid a_{1}, \ldots, a_{r}\right)\left\langle\prod_{j=1}^{s}\left(\frac{(1+t)^{\lambda_{j}}}{1+(1+t)^{\lambda_{j}}}\right)^{\mu_{j}} \mid x^{n-l}\right\rangle \\
& =\sum_{l=0}^{n}\left(\begin{array}{c}
n \\
l
\end{array}\right) \widehat{N}_{l}\left(y \mid a_{1}, \ldots, a_{r}\right)\left\langle\sum_{i=0}^{\infty} \hat{s}_{i}\left(\lambda_{1}, \ldots, \lambda_{r} ; \mu_{1}, \ldots, \mu_{r}\right) \frac{t^{i}}{i !} \mid x^{n-l}\right\rangle \\
& =\sum_{l=0}^{n}\left(\begin{array}{c}
n \\
l
\end{array}\right) \widehat{N}_{l}\left(y \mid a_{1}, \ldots, a_{r}\right) \hat{s}_{n-l}\left(\lambda_{1}, \ldots, \lambda_{r} ; \mu_{1}, \ldots, \mu_{r}\right) .
\end{aligned}
$$

Thus, we obtain (17).

Finally, we obtain

$$
\begin{aligned}
\widehat{\mathrm{NS}}_{n} & \left(y \mid a_{1}, \ldots, a_{r} ; \lambda_{1}, \ldots, \lambda_{s} ; \mu_{1}, \ldots, \mu_{s}\right) \\
& =\left\langle\left.\sum_{i=0}^{\infty} \widehat{\mathrm{NS}}_{i}\left(y \mid a_{1}, \ldots, a_{r} ; \lambda_{1}, \ldots, \lambda_{s} ; \mu_{1}, \ldots, \mu_{s}\right) \frac{t^{i}}{i !}\right|^{n}\right\rangle \\
& =\left\langle\prod_{i=1}^{r}\left(\frac{(1+t)^{a_{i}}-1}{(1+t)^{a_{i}} \ln (1+t)}\right) \prod_{j=1}^{s}\left(\frac{(1+t)^{\lambda_{j}}}{1+(1+t)^{\lambda_{j}}}\right)^{\mu_{j}}(1+t)^{y} \mid x^{n}\right\rangle \\
& \left\langle\prod_{i=1}^{r}\left(\frac{(1+t)^{a_{i}}-1}{(1+t)^{a_{i}} \ln (1+t)}\right) \mid \prod_{j=1}^{s}\left(\frac{(1+t)^{\lambda_{j}}}{1+(1+t)^{\lambda_{j}}}\right)^{\mu_{j}}(1+t)^{y} x^{n}\right\rangle \\
& =\left\langle\prod_{i=1}^{r}\left(\frac{(1+t)^{a_{i}}-1}{(1+t)^{a_{i}} \ln (1+t)}\right) \mid \sum_{l=0}^{\infty} \hat{s}_{l}\left(y \mid \lambda_{1}, \ldots, \lambda_{s} ; \mu_{1}, \ldots, \mu_{s}\right) \frac{t^{l}}{l !} x^{n}\right\rangle \\
& =\sum_{l=0}^{n} \hat{s}_{l}\left(y \mid \lambda_{1}, \ldots, \lambda_{s} ; \mu_{1}, \ldots, \mu_{s}\right)\left(\begin{array}{c}
n \\
l
\end{array}\right)\left\langle\prod_{i=1}^{r}\left(\frac{(1+t)^{a_{i}}-1}{(1+t)^{a_{i}} \ln (1+t)}\right) \mid x^{n-l}\right\rangle \\
& =\sum_{l=0}^{n} \hat{s}_{l}\left(y \mid \lambda_{1}, \ldots, \lambda_{s} ; \mu_{1}, \ldots, \mu_{s}\right)\left(\begin{array}{c}
n \\
l
\end{array}\right)\left\langle\sum_{i=0}^{\infty} \widehat{N}_{i}\left(a_{1}, \ldots, a_{r}\right) \frac{t^{i}}{i !} \mid x^{n-l}\right\rangle \\
& =\sum_{l=0}^{n}\left(\begin{array}{l}
n \\
l
\end{array}\right) \hat{s}_{l}\left(y \mid \lambda_{1}, \ldots, \lambda_{s} ; \mu_{1}, \ldots, \mu_{s}\right) \widehat{N}_{n-l}\left(a_{1}, \ldots, a_{r}\right) .
\end{aligned}
$$

Thus, we get the identity (18). 


\subsection{Sheffer identity}

\section{Theorem 2}

$$
\begin{aligned}
& \widehat{\mathrm{NS}}_{n}\left(x+y \mid a_{1}, \ldots, a_{r} ; \lambda_{1}, \ldots, \lambda_{s} ; \mu_{1}, \ldots, \mu_{s}\right) \\
& \quad=\sum_{l=0}^{n}\left(\begin{array}{l}
n \\
l
\end{array}\right) \widehat{\mathrm{NS}}_{l}\left(x \mid a_{1}, \ldots, a_{r} ; \lambda_{1}, \ldots, \lambda_{s} ; \mu_{1}, \ldots, \mu_{s}\right)(y)_{n-l} .
\end{aligned}
$$

Proof By (12) with

$$
\begin{aligned}
p_{n}(x) & =\prod_{i=1}^{r}\left(\frac{t e^{a_{i} t}}{e^{a_{i} t}-1}\right) \prod_{j=1}^{s}\left(\frac{1+e^{\lambda_{j} t}}{e^{\lambda_{j} t}}\right)^{\mu_{j}} \widehat{\mathrm{NS}}_{n}\left(x \mid a_{1}, \ldots, a_{r} ; \lambda_{1}, \ldots, \lambda_{s} ; \mu_{1}, \ldots, \mu_{s}\right) \\
& =(x)_{n} \sim\left(1, e^{t}-1\right),
\end{aligned}
$$

using (10), we have (21).

\subsection{Difference relations}

\section{Theorem 3}

$$
\begin{aligned}
& \widehat{\mathrm{NS}}_{n}\left(x+1 \mid a_{1}, \ldots, a_{r} ; \lambda_{1}, \ldots, \lambda_{s} ; \mu_{1}, \ldots, \mu_{s}\right)-\widehat{\mathrm{NS}}_{n}\left(x \mid a_{1}, \ldots, a_{r} ; \lambda_{1}, \ldots, \lambda_{s} ; \mu_{1}, \ldots, \mu_{s}\right) \\
& \quad=n \widehat{\mathrm{NS}}_{n-1}\left(x \mid a_{1}, \ldots, a_{r} ; \lambda_{1}, \ldots, \lambda_{s} ; \mu_{1}, \ldots, \mu_{s}\right) .
\end{aligned}
$$

Proof By (8) with (12), we get

$$
\begin{gathered}
\left(e^{t}-1\right) \widehat{\mathrm{NS}}_{n}\left(x \mid a_{1}, \ldots, a_{r} ; \lambda_{1}, \ldots, \lambda_{s} ; \mu_{1}, \ldots, \mu_{s}\right) \\
=n \widehat{\mathrm{NS}}_{n-1}\left(x \mid a_{1}, \ldots, a_{r} ; \lambda_{1}, \ldots, \lambda_{s} ; \mu_{1}, \ldots, \mu_{s}\right) .
\end{gathered}
$$

By (7), we have (22).

\subsection{Recurrence}

\section{Theorem 4}

$$
\begin{aligned}
& \widehat{\mathrm{NS}}_{n+1}\left(x \mid a_{1}, \ldots, a_{r} ; \lambda_{1}, \ldots, \lambda_{s} ; \mu_{1}, \ldots, \mu_{s}\right) \\
& =\left(x+\sum_{j=1}^{s} \mu_{j} \lambda_{j}-\sum_{i=1}^{r} a_{i}\right) \widehat{\mathrm{NS}}_{n}\left(x-1 \mid a_{1}, \ldots, a_{r} ; \lambda_{1}, \ldots, \lambda_{s} ; \mu_{1}, \ldots, \mu_{s}\right) \\
& \quad+2^{-\sum_{j=1}^{s} \mu_{j}} \sum_{m=0}^{n} \sum_{l=0}^{m} \frac{(-1)^{m+1}\left(\begin{array}{c}
m \\
l
\end{array}\right)}{l+1} S_{1}(n, m) E_{m-l}\left(\lambda_{1}, \ldots, \lambda_{s} ; \mu_{1}, \ldots, \mu_{s}\right) \\
& \quad \times\left(\sum_{i=1}^{r} a_{i} F_{l+1}\left(1-x \mid a_{1}, \ldots, a_{i-1}, a_{i+1}, \ldots, a_{r}\right)-r F_{l+1}\left(1-x \mid a_{1}, \ldots, a_{r}\right)\right) \\
& \quad+2^{-1-\sum_{j=1}^{s} \mu_{j}} \sum_{m=0}^{n} \sum_{l=0}^{m}(-1)^{m+1}\left(\begin{array}{c}
m \\
l
\end{array}\right) S_{1}(n, m) F_{m-l}\left(a_{1}, \ldots, a_{r}\right) \\
& \quad \times \sum_{i=1}^{s} \mu_{i} \lambda_{i} E_{l}\left(1-x \mid \lambda ; \mu+e_{i}\right) .
\end{aligned}
$$


Proof By applying

$$
s_{n+1}(x)=\left(x-\frac{g^{\prime}(t)}{g(t)}\right) \frac{1}{f^{\prime}(t)} s_{n}(x)
$$

[1, Corollary 3.7.2] with (12), we get

$$
\widehat{\mathrm{NS}}_{n+1}(x)=x \widehat{\mathrm{NS}}_{n}(x-1)-e^{-t} \frac{g^{\prime}(t)}{g(t)} \widehat{\mathrm{NS}}_{n}(x) .
$$

Observe that

$$
\begin{aligned}
\frac{g^{\prime}(t)}{g(t)}= & (\ln g(t))^{\prime} \\
& =\left(r \ln t+\left(\sum_{i=1}^{r} a_{i}\right) t-\sum_{i=1}^{r} \ln \left(e^{a_{i} t}-1\right)+\sum_{j=1}^{s} \mu_{j} \ln \left(1+e^{\lambda_{j} t}\right)-\left(\sum_{j=1}^{s} \mu_{j} \lambda_{j}\right) t\right)^{\prime} \\
& =\frac{r}{t}+\sum_{i=1}^{r} a_{i}-\sum_{i=1}^{r} \frac{a_{i} e^{a_{i} t}}{e^{a_{i} t}-1}+\sum_{j=1}^{s} \frac{\mu_{j} \lambda_{j} e^{\lambda_{j} t}}{1+e^{\lambda_{j} t}}-\sum_{j=1}^{s} \mu_{j} \lambda_{j} \\
& =\frac{r-\sum_{i=1}^{r} \frac{a_{i} t^{a_{i} t}}{e^{a_{i} t}-1}}{t}+\sum_{i=1}^{r} a_{i}+\sum_{j=1}^{s} \frac{\mu_{j} \lambda_{j} e^{\lambda_{j} t}}{1+e^{\lambda_{j} t}}-\sum_{j=1}^{s} \mu_{j} \lambda_{j},
\end{aligned}
$$

where

$$
r-\sum_{i=1}^{r} \frac{a_{i} t e^{a_{i} t}}{e^{a_{i} t}-1}=-\frac{1}{2}\left(\sum_{i=1}^{r} a_{i}\right) t+\cdots
$$

has the order at least one. Since from the proofs of (13) and (15)

$$
\begin{aligned}
\widehat{\mathrm{NS}}_{n}(x)= & 2^{-\sum_{j=1}^{s} \mu_{j}} \sum_{m=0}^{n}(-1)^{m} S_{1}(n, m) \\
& \times \sum_{l=0}^{m}(-1)^{l}\left(\begin{array}{c}
m \\
l
\end{array}\right) E_{m-l}\left(\lambda_{1}, \ldots, \lambda_{s} ; \mu_{1}, \ldots, \mu_{s}\right) \prod_{i=1}^{r}\left(\frac{e^{-a_{i} t}-1}{-t}\right) x^{l} \\
= & 2^{-\sum_{j=1}^{s} \mu_{j}} \sum_{m=0}^{n}(-1)^{m} S_{1}(n, m) \\
& \times \sum_{l=0}^{m}\left(\begin{array}{c}
m \\
l
\end{array}\right) F_{m-l}\left(a_{1}, \ldots, a_{r}\right)(-1)^{l} \prod_{j=1}^{s}\left(\frac{2}{1+e^{-\lambda_{j} t}}\right)^{\mu_{j}} x^{l},
\end{aligned}
$$

we have

$$
\begin{aligned}
& \frac{g^{\prime}(t)}{g(t)} \widehat{\mathrm{NS}}_{n}(x) \\
& \quad=\left(\sum_{i=1}^{r} a_{i}-\sum_{j=1}^{s} \mu_{j} \lambda_{j}\right) \widehat{\mathrm{NS}}_{n}(x)
\end{aligned}
$$




$$
\begin{aligned}
& +2^{-\sum_{j=1}^{s} \mu_{j}} \sum_{m=0}^{n}(-1)^{m} S_{1}(n, m) \sum_{l=0}^{m}(-1)^{l}\left(\begin{array}{c}
m \\
l
\end{array}\right) E_{m-l}\left(\lambda_{1}, \ldots, \lambda_{s} ; \mu_{1}, \ldots, \mu_{s}\right) \\
& \times \prod_{i=1}^{r}\left(\frac{e^{-a_{i} t}-1}{-t}\right) \frac{r-\sum_{i=1}^{r} \frac{a_{i} t}{1-e^{-a_{i} t}}}{t} x^{l} \\
& +2^{-\sum_{j=1}^{s} \mu_{j}} \sum_{m=0}^{n}(-1)^{m} S_{1}(n, m) \sum_{l=0}^{m}\left(\begin{array}{c}
m \\
l
\end{array}\right) F_{m-l}\left(a_{1}, \ldots, a_{r}\right)(-1)^{l} \\
& \times \sum_{i=1}^{s} \frac{\mu_{i} \lambda_{i}}{1+e^{-\lambda_{i} t}} \prod_{j=1}^{s}\left(\frac{2}{1+e^{-\lambda_{j} t}}\right)^{\mu_{j}} x^{l}
\end{aligned}
$$

The third term is equal to

$$
\begin{aligned}
& 2^{-\sum_{j=1}^{s} \mu_{j}} \sum_{m=0}^{n} \sum_{l=0}^{m}(-1)^{m}\left(\begin{array}{c}
m \\
l
\end{array}\right) S_{1}(n, m) F_{m-l}\left(a_{1}, \ldots, a_{r}\right)(-1)^{l} \\
& \quad \times \sum_{i=1}^{s} \frac{\mu_{i} \lambda_{i}}{2}(-1)^{l} E_{l}\left(-x \mid \lambda ; \mu+e_{i}\right) \\
& =2^{-1-\sum_{j=1}^{s} \mu_{j}} \sum_{m=0}^{n} \sum_{l=0}^{m}(-1)^{m}\left(\begin{array}{c}
m \\
l
\end{array}\right) S_{1}(n, m) F_{m-l}\left(a_{1}, \ldots, a_{r}\right) \\
& \quad \times \sum_{i=1}^{s} \mu_{i} \lambda_{i} E_{l}\left(-x \mid \lambda ; \mu+e_{i}\right),
\end{aligned}
$$

where $\lambda=\left(\lambda_{1}, \ldots, \lambda_{s}\right), \mu=\left(\mu_{1}, \ldots, \mu_{s}\right)$, and $e_{i}=(\underbrace{0, \ldots, 0}_{i-1}, 1, \underbrace{0, \ldots, 0}_{s-i})$. The second term is

$$
\begin{aligned}
& 2^{-\sum_{j=1}^{s} \mu_{j}} \sum_{m=0}^{n}(-1)^{m} S_{1}(n, m) \sum_{l=0}^{m} \frac{(-1)^{l}}{l+1}\left(\begin{array}{c}
m \\
l
\end{array}\right) E_{m-l}\left(\lambda_{1}, \ldots, \lambda_{s} ; \mu_{1}, \ldots, \mu_{s}\right) \\
& \times \prod_{i=1}^{r}\left(\frac{e^{-a_{i} t}-1}{-t}\right)\left(r-\sum_{i=1}^{r} \frac{a_{i} t}{1-e^{-a_{i} t}}\right) x^{l+1} \\
& =r 2^{-\sum_{j=1}^{s} \mu_{j}} \sum_{m=0}^{n}(-1)^{m} S_{1}(n, m) \sum_{l=0}^{m} \frac{(-1)^{l}}{l+1}\left(\begin{array}{c}
m \\
l
\end{array}\right) E_{m-l}\left(\lambda_{1}, \ldots, \lambda_{s} ; \mu_{1}, \ldots, \mu_{s}\right) \\
& \times \prod_{i=1}^{r}\left(\frac{e^{-a_{i} t}-1}{-t}\right) x^{l+1} \\
& -2^{-\sum_{j=1}^{s} \mu_{j}} \sum_{m=0}^{n}(-1)^{m} S_{1}(n, m) \sum_{l=0}^{m} \frac{(-1)^{l}}{l+1}\left(\begin{array}{c}
m \\
l
\end{array}\right) E_{m-l}\left(\lambda_{1}, \ldots, \lambda_{s} ; \mu_{1}, \ldots, \mu_{s}\right) \\
& \times \sum_{i=1}^{r} a_{i} \frac{-t}{e^{-a_{i} t}-1} \prod_{i=1}^{r}\left(\frac{e^{-a_{i} t}-1}{-t}\right) x^{l+1} \\
& =r 2^{-\sum_{j=1}^{s} \mu_{j}} \sum_{m=0}^{n}(-1)^{m} S_{1}(n, m) \sum_{l=0}^{m} \frac{(-1)^{l}}{l+1}\left(\begin{array}{c}
m \\
l
\end{array}\right) E_{m-l}\left(\lambda_{1}, \ldots, \lambda_{s} ; \mu_{1}, \ldots, \mu_{s}\right) \\
& \times(-1)^{l+1} F_{l+1}\left(-x \mid a_{1}, \ldots, a_{r}\right) \\
& -2^{-\sum_{j=1}^{s} \mu_{j}} \sum_{m=0}^{n}(-1)^{m} S_{1}(n, m) \sum_{l=0}^{m} \frac{(-1)^{l}}{l+1}\left(\begin{array}{c}
m \\
l
\end{array}\right) E_{m-l}\left(\lambda_{1}, \ldots, \lambda_{s} ; \mu_{1}, \ldots, \mu_{s}\right)
\end{aligned}
$$




$$
\begin{aligned}
& \times \sum_{i=1}^{r} a_{i}(-1)^{l+1} F_{l+1}\left(-x \mid a_{1}, \ldots, a_{i-1}, a_{i+1}, \ldots, a_{r}\right) \\
= & 2^{-\sum_{j=1}^{s} \mu_{j}} \sum_{m=0}^{n} \sum_{l=0}^{m} \frac{(-1)^{m+1}\left(\begin{array}{c}
m \\
l
\end{array}\right)}{l+1} S_{1}(n, m) E_{m-l}\left(\lambda_{1}, \ldots, \lambda_{s} ; \mu_{1}, \ldots, \mu_{s}\right) \\
& \times\left(r F_{l+1}\left(-x \mid a_{1}, \ldots, a_{r}\right)-\sum_{i=1}^{r} a_{i} F_{l+1}\left(-x \mid a_{1}, \ldots, a_{i-1}, a_{i+1}, \ldots, a_{r}\right)\right) .
\end{aligned}
$$

Therefore, we obtain

$$
\begin{aligned}
& \widehat{\mathrm{NS}}_{n+1}(x) \\
& =\left(x+\sum_{j=1}^{s} \mu_{j} \lambda_{j}-\sum_{i=1}^{r} a_{i}\right) \widehat{\mathrm{NS}}_{n}(x-1) \\
& \quad+2^{-\sum_{j=1}^{s} \mu_{j}} \sum_{m=0}^{n} \sum_{l=0}^{m} \frac{(-1)^{m+1}\left(\begin{array}{c}
m \\
l
\end{array}\right)}{l+1} S_{1}(n, m) E_{m-l}\left(\lambda_{1}, \ldots, \lambda_{s} ; \mu_{1}, \ldots, \mu_{s}\right) \\
& \quad \times\left(\sum_{i=1}^{r} a_{i} F_{l+1}\left(1-x \mid a_{1}, \ldots, a_{i-1}, a_{i+1}, \ldots, a_{r}\right)-r F_{l+1}\left(1-x \mid a_{1}, \ldots, a_{r}\right)\right) \\
& \quad+2^{-1-\sum_{j=1}^{s} \mu_{j}} \sum_{m=0}^{n} \sum_{l=0}^{m}(-1)^{m+1}\left(\begin{array}{c}
m \\
l
\end{array}\right) S_{1}(n, m) F_{m-l}\left(a_{1}, \ldots, a_{r}\right) \\
& \quad \times \sum_{i=1}^{s} \mu_{i} \lambda_{i} E_{l}\left(1-x \mid \lambda ; \mu+e_{i}\right),
\end{aligned}
$$

which is the identity (23).

\subsection{Differentiation}

\section{Theorem 5}

$$
\begin{aligned}
& \frac{d}{d x} \widehat{\mathrm{NS}}_{n}\left(x \mid a_{1}, \ldots, a_{r} ; \lambda_{1}, \ldots, \lambda_{s} ; \mu_{1}, \ldots, \mu_{s}\right) \\
& \quad=n ! \sum_{l=0}^{n-1} \frac{(-1)^{n-l-1}}{l !(n-l)} \widehat{\mathrm{NS}}_{l}\left(x \mid a_{1}, \ldots, a_{r} ; \lambda_{1}, \ldots, \lambda_{s} ; \mu_{1}, \ldots, \mu_{s}\right) .
\end{aligned}
$$

Proof We shall use

$$
\frac{d}{d x} s_{n}(x)=\sum_{l=0}^{n-1}\left(\begin{array}{l}
n \\
l
\end{array}\right)\left\langle\bar{f}(t) \mid x^{n-l}\right\rangle s_{l}(x)
$$

(cf. [1, Theorem 2.3.12]). Since

$$
\begin{aligned}
\left\langle\bar{f}(t) \mid x^{n-l}\right\rangle & =\left\langle\ln (1+t) \mid x^{n-l}\right\rangle \\
& =\left\langle\sum_{m=1}^{\infty} \frac{(-1)^{m-1} t^{m}}{m} \mid x^{n-l}\right\rangle
\end{aligned}
$$




$$
\begin{aligned}
& =\sum_{m=1}^{n-l} \frac{(-1)^{m-1}}{m}\left\langle t^{m} \mid x^{n-l}\right\rangle \\
& =\sum_{m=1}^{n-l} \frac{(-1)^{m-1}}{m}(n-l) ! \delta_{m, n-l} \\
& =(-1)^{n-l-1}(n-l-1) !
\end{aligned}
$$

with (12), we have

$$
\begin{aligned}
& \frac{d}{d x} \widehat{\mathrm{NS}}_{n}\left(x \mid a_{1}, \ldots, a_{r} ; \lambda_{1}, \ldots, \lambda_{s} ; \mu_{1}, \ldots, \mu_{s}\right) \\
& \quad=\sum_{l=0}^{n-1}\left(\begin{array}{l}
n \\
l
\end{array}\right)(-1)^{n-l-1}(n-l-1) ! \widehat{\mathrm{NS}}_{l}\left(x \mid a_{1}, \ldots, a_{r} ; \lambda_{1}, \ldots, \lambda_{s} ; \mu_{1}, \ldots, \mu_{s}\right) \\
& \quad=n ! \sum_{l=0}^{n-1} \frac{(-1)^{n-l-1}}{l !(n-l)} \widehat{\mathrm{NS}}_{l}\left(x \mid a_{1}, \ldots, a_{r} ; \lambda_{1}, \ldots, \lambda_{s} ; \mu_{1}, \ldots, \mu_{s}\right)
\end{aligned}
$$

which is the identity (25).

\subsection{One more relation}

The classical Cauchy numbers of the first kind $c_{n}$ are defined by

$$
\frac{t}{\ln (1+t)}=\sum_{n=0}^{\infty} c_{n} \frac{t^{n}}{n !}
$$

(see e.g. $[3,4])$.

\section{Theorem 6}

$$
\begin{aligned}
& \widehat{\mathrm{NS}}_{n}(x \mid a ; \lambda ; \mu) \\
& =\left(x-\sum_{i=1}^{r} a_{i}\right) \widehat{\mathrm{NS}}_{n-1}(x-1 \mid a ; \lambda ; \mu)+\sum_{j=1}^{s} \lambda_{j} \mu_{j} \widehat{\mathrm{NS}}_{n-1}\left(x-\lambda_{j}-1 \mid a ; \lambda ; \mu+e_{j}\right) \\
& \quad+\frac{1}{n} \sum_{l=0}^{n}\left(\begin{array}{l}
n \\
l
\end{array}\right) c_{l}\left(\sum_{i=1}^{r} a_{i} \widehat{\mathrm{NS}}_{n-l}\left(x-1 \mid a_{1}, \ldots, a_{i-1}, a_{i+1}, \ldots, a_{r} ; \lambda ; \mu\right)\right. \\
& \left.\quad-r \widehat{\mathrm{NS}}_{n-l}(x-1 \mid a ; \lambda ; \mu)\right) .
\end{aligned}
$$

Proof For $n \geq 1$, we have

$$
\begin{aligned}
\widehat{\mathrm{NS}}_{n} & \left(y \mid a_{1}, \ldots, a_{r} ; \lambda_{1}, \ldots, \lambda_{s} ; \mu_{1}, \ldots, \mu_{s}\right) \\
& =\left\langle\sum_{l=0}^{\infty} \widehat{\mathrm{NS}}_{l}\left(y \mid a_{1}, \ldots, a_{r} ; \lambda_{1}, \ldots, \lambda_{s} ; \mu_{1}, \ldots, \mu_{s}\right) \frac{t^{l}}{l !} \mid x^{n}\right\rangle \\
& =\left\langle\prod_{i=1}^{r}\left(\frac{(1+t)^{a_{i}}-1}{(1+t)^{a_{i}} \ln (1+t)}\right) \prod_{j=1}^{s}\left(\frac{(1+t)^{\lambda_{j}}}{1+(1+t)^{\lambda_{j}}}\right)^{\mu_{j}}(1+t)^{y} \mid x^{n}\right\rangle
\end{aligned}
$$




$$
\begin{aligned}
= & \left\langle\partial_{t}\left(\prod_{i=1}^{r}\left(\frac{(1+t)^{a_{i}}-1}{(1+t)^{a_{i}} \ln (1+t)}\right) \prod_{j=1}^{s}\left(\frac{(1+t)^{\lambda_{j}}}{1+(1+t)^{\lambda_{j}}}\right)^{\mu_{j}}(1+t)^{y}\right) \mid x^{n-1}\right\rangle \\
= & \left\langle\left(\partial_{t} \prod_{i=1}^{r}\left(\frac{(1+t)^{a_{i}}-1}{(1+t)^{a_{i}} \ln (1+t)}\right)\right) \prod_{j=1}^{s}\left(\frac{(1+t)^{\lambda_{j}}}{1+(1+t)^{\lambda_{j}}}\right)^{\mu_{j}}(1+t)^{y} \mid x^{n-1}\right\rangle \\
& +\left\langle\prod_{i=1}^{r}\left(\frac{(1+t)^{a_{i}}-1}{(1+t)^{a_{i}} \ln (1+t)}\right)\left(\partial_{t} \prod_{j=1}^{s}\left(\frac{(1+t)^{\lambda_{j}}}{1+(1+t)^{\lambda_{j}}}\right)^{\mu_{j}}\right\rangle(1+t)^{y} \mid x^{n-1}\right\rangle \\
& +\left\langle\prod_{i=1}^{r}\left(\frac{(1+t)^{a_{i}}-1}{(1+t)^{a_{i}} \ln (1+t)}\right) \prod_{j=1}^{s}\left(\frac{(1+t)^{\lambda_{j}}}{1+(1+t)^{\lambda_{j}}}\right)^{\mu_{j}}\left(\partial_{t}(1+t)^{y}\right) \mid x^{n-1}\right\rangle .
\end{aligned}
$$

The third term is

$$
\begin{aligned}
& y\left\langle\prod_{i=1}^{r}\left(\frac{(1+t)^{a_{i}}-1}{(1+t)^{a_{i}} \ln (1+t)}\right) \prod_{j=1}^{s}\left(\frac{(1+t)^{\lambda_{j}}}{1+(1+t)^{\lambda_{j}}}\right)^{\mu_{j}}(1+t)^{y-1} \mid x^{n-1}\right\rangle \\
& =y \widehat{\mathrm{NS}}_{n-1}\left(y-1 \mid a_{1}, \ldots, a_{r} ; \lambda_{1}, \ldots, \lambda_{s} ; \mu_{1}, \ldots, \mu_{s}\right) .
\end{aligned}
$$

Since

$$
\begin{aligned}
\partial_{t} & \prod_{i=1}^{r}\left(\frac{(1+t)^{a_{i}}-1}{(1+t)^{a_{i}} \ln (1+t)}\right) \\
= & \frac{1}{1+t} \prod_{i=1}^{r}\left(\frac{(1+t)^{a_{i}}-1}{(1+t)^{a_{i}} \ln (1+t)}\right) \frac{\sum_{v=1}^{r}\left(\frac{a_{v} t(1+t)^{a_{\nu}}}{(1+t)^{a_{\nu}-1}}-\frac{t}{\ln (1+t)}\right)}{t} \\
& -\frac{1}{1+t} \prod_{i=1}^{r}\left(\frac{(1+t)^{a_{i}}-1}{(1+t)^{a_{i}} \ln (1+t)}\right)\left(\sum_{l=1}^{r} a_{l}\right),
\end{aligned}
$$

with

$$
\sum_{\nu=1}^{r}\left(\frac{a_{\nu} t(1+t)^{a_{\nu}}}{(1+t)^{a_{\nu}}-1}-\frac{t}{\ln (1+t)}\right)
$$

having order $\geq 1$, the first term is

$$
\begin{aligned}
\left\langle\prod_{i=1}^{r}\right. & \left(\frac{(1+t)^{a_{i}}-1}{(1+t)^{a_{i}} \ln (1+t)}\right) \prod_{j=1}^{s}\left(\frac{(1+t)^{\lambda_{j}}}{1+(1+t)^{\lambda_{j}}}\right)^{\mu_{j}}(1+t)^{y-1}\left|\frac{\sum_{v=1}^{r}\left(\frac{a_{v} t(1+t)^{a_{\nu}}}{(1+t)^{a_{\nu}}-1}-\frac{t}{t}\right)}{\ln (1+t)} x^{n-1}\right\rangle \\
& -\left(\sum_{l=1}^{r} a_{l}\right)\left\langle\prod_{i=1}^{r}\left(\frac{(1+t)^{a_{i}}-1}{(1+t)^{a_{i}} \ln (1+t)}\right) \prod_{j=1}^{s}\left(\frac{(1+t)^{\lambda_{j}}}{1+(1+t)^{\lambda_{j}}}\right)^{\mu_{j}}(1+t)^{y-1} \mid x^{n-1}\right\rangle \\
= & -\left(\sum_{l=1}^{r} a_{l}\right) \widehat{\mathrm{NS}}_{n-1}(y-1)+\frac{1}{n}\left\langle\prod_{i=1}^{r}\left(\frac{(1+t)^{a_{i}}-1}{(1+t)^{a_{i}} \ln (1+t)}\right) \prod_{j=1}^{s}\left(\frac{(1+t)^{\lambda_{j}}}{1+(1+t)^{\lambda_{j}}}\right)^{\mu_{j}}\right. \\
& \times(1+t)^{y-1}\left|\sum_{\nu=1}^{r}\left(\frac{a_{\nu} t(1+t)^{a_{\nu}}}{(1+t)^{a_{\nu}}-1}-\frac{t}{\ln (1+t)}\right) x^{n}\right\rangle \\
= & -\left(\sum_{l=1}^{r} a_{l}\right) \widehat{\mathrm{NS}}_{n-1}(y-1)
\end{aligned}
$$




$$
\begin{aligned}
& +\frac{1}{n}\left(\sum _ { \nu = 1 } ^ { r } a _ { \nu } \left\langle\frac{(1+t)^{a_{\nu}} \ln (1+t)}{(1+t)^{a_{\nu}}-1} \prod_{i=1}^{r}\left(\frac{(1+t)^{a_{i}}-1}{(1+t)^{a_{i}} \ln (1+t)}\right) \prod_{j=1}^{s}\left(\frac{(1+t)^{\lambda_{j}}}{1+(1+t)^{\lambda_{j}}}\right)^{\mu_{j}}\right.\right. \\
& \times(1+t)^{y-1}\left|\frac{t}{\ln (1+t)} x^{n}\right\rangle \\
& \left.-r\left\langle\prod_{i=1}^{r}\left(\frac{(1+t)^{a_{i}}-1}{(1+t)^{a_{i}} \ln (1+t)}\right) \prod_{j=1}^{s}\left(\frac{(1+t)^{\lambda_{j}}}{1+(1+t)^{\lambda_{j}}}\right)^{\mu_{j}}(1+t)^{y-1} \mid \frac{t}{\ln (1+t)} x^{n}\right\rangle\right) \\
& =-\left(\sum_{l=1}^{r} a_{l}\right) \widehat{\mathrm{NS}}_{n-1}(y-1) \\
& +\frac{1}{n}\left(\sum _ { \nu = 1 } ^ { r } a _ { \nu } \left\langle\frac{(1+t)^{a_{\nu}} \ln (1+t)}{(1+t)^{a_{\nu}}-1} \prod_{i=1}^{r}\left(\frac{(1+t)^{a_{i}}-1}{(1+t)^{a_{i}} \ln (1+t)}\right) \prod_{j=1}^{s}\left(\frac{(1+t)^{\lambda_{j}}}{1+(1+t)^{\lambda_{j}}}\right)^{\mu_{j}}\right.\right. \\
& \times(1+t)^{y-1}\left|\sum_{l=0}^{\infty} c_{l} \frac{t^{l}}{l !} x^{n}\right\rangle \\
& -r\left(\prod_{i=1}^{r}\left(\frac{(1+t)^{a_{i}}-1}{(1+t)^{a_{i}} \ln (1+t)}\right) \prod_{j=1}^{s}\left(\frac{(1+t)^{\lambda_{j}}}{1+(1+t)^{\lambda_{j}}}\right)^{\mu_{j}}(1+t)^{y-1}\left|\sum_{l=0}^{\infty} c_{l} \frac{t^{l}}{l !} x^{n}\right\rangle\right) \\
& =-\left(\sum_{l=1}^{r} a_{l}\right) \widehat{\mathrm{NS}}_{n-1}(y-1) \\
& +\frac{1}{n}\left(\sum_{\nu=1}^{r} \sum_{l=0}^{n}\left(\begin{array}{l}
n \\
l
\end{array}\right) a_{\nu} c_{l} \widehat{N S}_{n-l}\left(y-1 \mid a_{1}, \ldots, a_{v-1}, a_{v+1}, \ldots, a_{r} ; \lambda ; \mu\right)\right. \\
& \left.-r \sum_{l=0}^{n}\left(\begin{array}{l}
n \\
l
\end{array}\right) c_{l} \widehat{\mathrm{NS}}_{n-l}(y-1 \mid a ; \lambda ; \mu)\right) \\
& =-\left(\sum_{\nu=1}^{r} a_{\nu}\right) \widehat{\mathrm{NS}}_{n-1}(y-1) \\
& +\frac{1}{n} \sum_{l=0}^{n}\left(\begin{array}{l}
n \\
l
\end{array}\right) c_{l}\left(\sum_{\nu=1}^{r} a_{\nu} \widehat{\mathrm{NS}}_{n-l}\left(y-1 \mid a_{1}, \ldots, a_{\nu-1}, a_{\nu+1}, \ldots, a_{r} ; \lambda ; \mu\right)\right. \\
& \left.-r \widehat{\mathrm{NS}}_{n-l}(y-1 \mid a ; \lambda ; \mu)\right) \text {. }
\end{aligned}
$$

Since

$$
\partial_{t} \prod_{j=1}^{s}\left(\frac{(1+t)^{\lambda_{j}}}{1+(1+t)^{\lambda_{j}}}\right)^{\mu_{j}}=\sum_{l=1}^{s} \lambda_{l} \mu_{l}(1+t)^{-\lambda_{l}-1}\left(\frac{(1+t)^{\lambda_{l}}}{1+(1+t)^{\lambda_{l}}}\right) \prod_{j=1}^{s}\left(\frac{(1+t)^{\lambda_{j}}}{1+(1+t)^{\lambda_{j}}}\right)^{\mu_{j}},
$$

the second term is

$$
\begin{aligned}
& \sum_{l=1}^{s} \lambda_{l} \mu_{l}\left\langle\prod_{i=1}^{r}\left(\frac{(1+t)^{a_{i}}-1}{(1+t)^{a_{i}} \ln (1+t)}\right)\left(\frac{(1+t)^{\lambda_{l}}}{1+(1+t)^{\lambda_{l}}}\right) \prod_{j=1}^{s}\left(\frac{(1+t)^{\lambda_{j}}}{1+(1+t)^{\lambda_{j}}}\right)^{\mu_{j}}(1+t)^{y-\lambda_{l}-1} \mid x^{n-1}\right\rangle \\
& \quad=\sum_{l=1}^{s} \lambda_{l} \mu_{l} \widehat{\mathrm{NS}}_{n-1}\left(y-\lambda_{l}-1 \mid a ; \lambda ; \mu+e_{l}\right) .
\end{aligned}
$$


Therefore, we obtain

$$
\begin{aligned}
& \widehat{\mathrm{NS}}_{n}(x \mid a ; \lambda ; \mu) \\
& =\left(x-\sum_{i=1}^{r} a_{i}\right) \widehat{\mathrm{NS}}_{n-1}(x-1 \mid a ; \lambda ; \mu)+\sum_{j=1}^{s} \lambda_{j} \mu_{j} \widehat{\mathrm{NS}}_{n-1}\left(x-\lambda_{j}-1 \mid a ; \lambda ; \mu+e_{j}\right) \\
& \quad+\frac{1}{n} \sum_{l=0}^{n}\left(\begin{array}{l}
n \\
l
\end{array}\right) c_{l}\left(\sum_{i=1}^{r} a_{i} \widehat{\mathrm{NS}}_{n-l}\left(x-1 \mid a_{1}, \ldots, a_{i-1}, a_{i+1}, \ldots, a_{r} ; \lambda ; \mu\right)\right. \\
& \left.\quad-r \widehat{\mathrm{NS}}_{n-l}(x-1 \mid a ; \lambda ; \mu)\right)
\end{aligned}
$$

which is the identity (26).

\subsection{A relation involving the Stirling numbers of the first kind}

Theorem 7 For $n-1 \geq m \geq 1$, we have

$$
\begin{aligned}
\sum_{l=0}^{n-m}\left(\begin{array}{l}
n \\
l
\end{array}\right) S_{1}(n-l, m) \widehat{\mathrm{NS}}_{l}(a ; \lambda ; \mu) \\
=-\left(\sum_{i=1}^{r} a_{i}\right) \sum_{l=0}^{n-m-1}\left(\begin{array}{c}
n-1 \\
l
\end{array}\right) S_{1}(n-l-1, m) \widehat{\mathrm{NS}}_{l}(-1 \mid a ; \lambda ; \mu) \\
+\frac{1}{n} \sum_{k=0}^{n-m} \sum_{l=k}^{n-m}\left(\begin{array}{l}
n \\
l
\end{array}\right)\left(\begin{array}{l}
l \\
k
\end{array}\right) S_{1}(n-l, m) c_{l-k} \\
+\left(\sum_{i=1}^{r} a_{i} \widehat{\mathrm{NS}}_{k}\left(-1 \mid a_{1}, \ldots, a_{i-1}, a_{i+1}, \ldots, a_{r} ; \lambda ; \mu\right)-r \widehat{\mathrm{NS}}_{k}(-1 \mid a ; \lambda ; \mu)\right) \\
+\sum_{j=1}^{s} \lambda_{j} \mu_{j} \sum_{l=0}^{n-m-1}\left(\begin{array}{c}
n-1 \\
l
\end{array}\right) S_{1}(n-l-1, m) \widehat{\mathrm{NS}}_{l}\left(-\lambda j-1 \mid a ; \lambda ; \mu+e_{j}\right) \\
+\sum_{l=0}^{n-m}\left(\begin{array}{c}
n-1 \\
l
\end{array}\right) S_{1}(n-l-1, m-1) \widehat{\mathrm{NS}}_{l}(-1 \mid a ; \lambda ; \mu) .
\end{aligned}
$$

Proof We shall compute

$$
\left\langle\prod_{i=1}^{r}\left(\frac{(1+t)^{a_{i}}-1}{(1+t)^{a_{i}} \ln (1+t)}\right) \prod_{j=1}^{s}\left(\frac{(1+t)^{\lambda_{j}}}{1+(1+t)^{\lambda_{j}}}\right)^{\mu_{j}}(\ln (1+t))^{m} \mid x^{n}\right\rangle
$$

in two different ways. On the one hand, it is equal to

$$
\begin{aligned}
& \left\langle\prod_{i=1}^{r}\left(\frac{(1+t)^{a_{i}}-1}{(1+t)^{a_{i}} \ln (1+t)}\right) \prod_{j=1}^{s}\left(\frac{(1+t)^{\lambda_{j}}}{1+(1+t)^{\lambda_{j}}}\right)^{\mu_{j}} \mid(\ln (1+t))^{m} x^{n}\right\rangle \\
& \quad=\left\langle\prod_{i=1}^{r}\left(\frac{(1+t)^{a_{i}}-1}{(1+t)^{a_{i}} \ln (1+t)}\right) \prod_{j=1}^{s}\left(\frac{(1+t)^{\lambda_{j}}}{1+(1+t)^{\lambda_{j}}}\right)^{\mu_{j}} \mid m ! \sum_{l=m}^{\infty} S_{1}(l, m) \frac{t^{l}}{l !} x^{n}\right\rangle \\
& \quad=m ! \sum_{l=m}^{n}\left(\begin{array}{l}
n \\
l
\end{array}\right) S_{1}(l, m)\left\langle\prod_{i=1}^{r}\left(\frac{(1+t)^{a_{i}}-1}{(1+t)^{a_{i}} \ln (1+t)}\right) \prod_{j=1}^{s}\left(\frac{(1+t)^{\lambda_{j}}}{1+(1+t)^{\lambda_{j}}}\right)^{\mu_{j}} \mid x^{n-l}\right\rangle
\end{aligned}
$$




$$
\begin{aligned}
& =m ! \sum_{l=m}^{n}\left(\begin{array}{l}
n \\
l
\end{array}\right) S_{1}(l, m)\left\langle\sum_{i=0}^{\infty} \widehat{\mathrm{NS}}_{i}\left(a_{1}, \ldots, a_{r} ; \lambda_{1}, \ldots, \lambda_{s} ; \mu_{1}, \ldots, \mu_{s}\right) \frac{t^{i}}{i !} \mid x^{n-l}\right\rangle \\
& =m ! \sum_{l=m}^{n}\left(\begin{array}{l}
n \\
l
\end{array}\right) S_{1}(l, m) \widehat{\mathrm{NS}}_{n-l}\left(a_{1}, \ldots, a_{r} ; \lambda_{1}, \ldots, \lambda_{s} ; \mu_{1}, \ldots, \mu_{s}\right) \\
& =m ! \sum_{l=0}^{n-m}\left(\begin{array}{l}
n \\
l
\end{array}\right) S_{1}(n-l, m) \widehat{\mathrm{NS}}_{l}\left(a_{1}, \ldots, a_{r} ; \lambda_{1}, \ldots, \lambda_{s} ; \mu_{1}, \ldots, \mu_{s}\right) .
\end{aligned}
$$

On the other hand, it is equal to

$$
\begin{aligned}
\left\langle\partial_{t}\right. & \left(\prod_{i=1}^{r}\left(\frac{(1+t)^{a_{i}}-1}{(1+t)^{a_{i}} \ln (1+t)}\right) \prod_{j=1}^{s}\left(\frac{(1+t)^{\lambda_{j}}}{1+(1+t)^{\lambda_{j}}}\right)^{\mu_{j}}(\ln (1+t))^{m}\right)\left|x^{n-1}\right\rangle \\
= & \left\langle\left(\partial_{t} \prod_{i=1}^{r}\left(\frac{(1+t)^{a_{i}}-1}{(1+t)^{a_{i}} \ln (1+t)}\right)\right) \prod_{j=1}^{s}\left(\frac{(1+t)^{\lambda_{j}}}{1+(1+t)^{\lambda_{j}}}\right)^{\mu_{j}}(\ln (1+t))^{m} \mid x^{n-1}\right\rangle \\
& +\left\langle\prod_{i=1}^{r}\left(\frac{(1+t)^{a_{i}}-1}{(1+t)^{a_{i}} \ln (1+t)}\right)\left(\partial_{t} \prod_{j=1}^{s}\left(\frac{(1+t)^{\lambda_{j}}}{1+(1+t)^{\lambda_{j}}}\right)^{\mu_{j}}\right)(\ln (1+t))^{m} \mid x^{n-1}\right\rangle \\
& +\left\langle\prod_{i=1}^{r}\left(\frac{(1+t)^{a_{i}}-1}{(1+t)^{a_{i}} \ln (1+t)}\right) \prod_{j=1}^{s}\left(\frac{(1+t)^{\lambda_{j}}}{1+(1+t)^{\lambda_{j}}}\right)^{\mu_{j}}\left(\partial_{t}(\ln (1+t))^{m}\right) \mid x^{n-1}\right\rangle .
\end{aligned}
$$

The third term of (28) is equal to

$$
\begin{aligned}
& m\left\langle\prod_{i=1}^{r}\left(\frac{(1+t)^{a_{i}}-1}{(1+t)^{a_{i}} \ln (1+t)}\right) \prod_{j=1}^{s}\left(\frac{(1+t)^{\lambda_{j}}}{1+(1+t)^{\lambda_{j}}}\right)^{\mu_{j}}(1+t)^{-1} \mid(\ln (1+t))^{m-1} x^{n-1}\right\rangle \\
& =m\left\langle\prod_{i=1}^{r}\left(\frac{(1+t)^{a_{i}}-1}{(1+t)^{a_{i}} \ln (1+t)}\right) \prod_{j=1}^{s}\left(\frac{(1+t)^{\lambda_{j}}}{1+(1+t)^{\lambda_{j}}}\right)^{\mu_{j}}\right. \\
& \times(1+t)^{-1}\left|(m-1) ! \sum_{l=m-1}^{\infty} S_{1}(l, m-1) \frac{t^{l}}{l !} x^{n-1}\right\rangle \\
& =m ! \sum_{l=m-1}^{n-1}\left(\begin{array}{c}
n-1 \\
l
\end{array}\right) S_{1}(l, m-1) \\
& \times\left\langle\prod_{i=1}^{r}\left(\frac{(1+t)^{a_{i}}-1}{(1+t)^{a_{i}} \ln (1+t)}\right) \prod_{j=1}^{s}\left(\frac{(1+t)^{\lambda_{j}}}{1+(1+t)^{\lambda_{j}}}\right)^{\mu_{j}}(1+t)^{-1} \mid x^{n-1-l}\right\rangle \\
& =m ! \sum_{l=m-1}^{n-1}\left(\begin{array}{c}
n-1 \\
l
\end{array}\right) S_{1}(l, m-1) \\
& \times \widehat{\mathrm{NS}}_{n-1-l}\left(-1 \mid a_{1}, \ldots, a_{r} ; \lambda_{1}, \ldots, \lambda_{s} ; \mu_{1}, \ldots, \mu_{s}\right) \\
& =m ! \sum_{l=0}^{n-m}\left(\begin{array}{c}
n-1 \\
l
\end{array}\right) S_{1}(n-l-1, m-1) \\
& \times \widehat{\mathrm{NS}}_{l}\left(-1 \mid a_{1}, \ldots, a_{r} ; \lambda_{1}, \ldots, \lambda_{s} ; \mu_{1}, \ldots, \mu_{s}\right) .
\end{aligned}
$$


Since

$$
\begin{aligned}
\partial_{t} & \prod_{i=1}^{r}\left(\frac{(1+t)^{a_{i}}-1}{(1+t)^{a_{i}} \ln (1+t)}\right) \\
= & \frac{1}{1+t} \prod_{i=1}^{r}\left(\frac{(1+t)^{a_{i}}-1}{(1+t)^{a_{i}} \ln (1+t)}\right) \frac{\sum_{\nu=1}^{r}\left(\frac{a_{v} t(1+t)^{a_{\nu}}}{(1+t)^{a_{\nu}}-1}-\frac{t}{\ln (1+t)}\right)}{t} \\
& -\frac{1}{1+t} \prod_{i=1}^{r}\left(\frac{(1+t)^{a_{i}}-1}{(1+t)^{a_{i}} \ln (1+t)}\right)\left(\sum_{\nu=1}^{r} a_{\nu}\right),
\end{aligned}
$$

the first term of (28) is equal to

$$
\begin{aligned}
& \frac{1}{n}\left\langle\prod_{i=1}^{r}\left(\frac{(1+t)^{a_{i}}-1}{(1+t)^{a_{i}} \ln (1+t)}\right) \prod_{j=1}^{s}\left(\frac{(1+t)^{\lambda_{j}}}{1+(1+t)^{\lambda_{j}}}\right)^{\mu_{j}}\right. \\
& \times(1+t)^{-1} \sum_{\nu=1}^{r}\left(\frac{a_{v} t(1+t)^{a_{v}}}{(1+t)^{a_{v}}-1}-\frac{t}{\ln (1+t)}\right)\left|(\ln (1+t))^{m} x^{n}\right\rangle \\
& -\left(\sum_{\nu=1}^{r} a_{\nu}\right)\left\langle\prod_{i=1}^{r}\left(\frac{(1+t)^{a_{i}}-1}{(1+t)^{a_{i}} \ln (1+t)}\right) \prod_{j=1}^{s}\left(\frac{(1+t)^{\lambda_{j}}}{1+(1+t)^{\lambda_{j}}}\right)^{\mu_{j}}\right. \\
& \times(1+t)^{-1}\left|(\ln (1+t))^{m} x^{n-1}\right\rangle \\
& =\frac{1}{n}\left\langle\prod_{i=1}^{r}\left(\frac{(1+t)^{a_{i}}-1}{(1+t)^{a_{i}} \ln (1+t)}\right) \prod_{j=1}^{s}\left(\frac{(1+t)^{\lambda_{j}}}{1+(1+t)^{\lambda_{j}}}\right)^{\mu_{j}}(1+t)^{-1}\right. \\
& \times \sum_{\nu=1}^{r}\left(\frac{a_{\nu} t(1+t)^{a_{\nu}}}{(1+t)^{a_{\nu}}-1}-\frac{t}{\ln (1+t)}\right)\left|m ! \sum_{l=m}^{\infty} S_{1}(l, m) \frac{t^{l}}{l !} x^{n}\right\rangle \\
& -\left(\sum_{\nu=1}^{r} a_{\nu}\right)\left\langle\prod_{i=1}^{r}\left(\frac{(1+t)^{a_{i}}-1}{(1+t)^{a_{i}} \ln (1+t)}\right) \prod_{j=1}^{s}\left(\frac{(1+t)^{\lambda_{j}}}{1+(1+t)^{\lambda_{j}}}\right)^{\mu_{j}}\right. \\
& \times(1+t)^{-1}\left|m ! \sum_{l=m}^{\infty} S_{1}(l, m) \frac{t^{l}}{l !} x^{n-1}\right\rangle \\
& =\frac{m !}{n} \sum_{l=m}^{n}\left(\begin{array}{l}
n \\
l
\end{array}\right) S_{1}(l, m)\left\langle\prod_{i=1}^{r}\left(\frac{(1+t)^{a_{i}}-1}{(1+t)^{a_{i}} \ln (1+t)}\right) \prod_{j=1}^{s}\left(\frac{(1+t)^{\lambda_{j}}}{1+(1+t)^{\lambda_{j}}}\right)^{\mu_{j}}\right. \\
& \times(1+t)^{-1} \sum_{\nu=1}^{r}\left(\frac{a_{\nu} t(1+t)^{a_{\nu}}}{(1+t)^{a_{\nu}}-1}-\frac{t}{\ln (1+t)}\right)\left|x^{n-l}\right\rangle \\
& -m !\left(\sum_{\nu=1}^{r} a_{\nu}\right) \sum_{l=m}^{n-1}\left(\begin{array}{c}
n-1 \\
l
\end{array}\right) S_{1}(l, m) \\
& \times\left\langle\prod_{i=1}^{r}\left(\frac{(1+t)^{a_{i}}-1}{(1+t)^{a_{i}} \ln (1+t)}\right) \prod_{j=1}^{s}\left(\frac{(1+t)^{\lambda_{j}}}{1+(1+t)^{\lambda_{j}}}\right)^{\mu_{j}}(1+t)^{-1} \mid x^{n-l-1}\right\rangle \\
& =-m !\left(\sum_{\nu=1}^{r} a_{v}\right) \sum_{l=m}^{n-1}\left(\begin{array}{c}
n-1 \\
l
\end{array}\right) S_{1}(l, m) \widehat{\mathrm{NS}}_{n-l-1}(-1)
\end{aligned}
$$


Kim et al. Journal of Inequalities and Applications 2014, 2014:376

Page 19 of 23

http://www.journalofinequalitiesandapplications.com/content/2014/1/376

$$
\begin{aligned}
& +\frac{m !}{n} \sum_{l=m}^{n}\left(\begin{array}{l}
n \\
l
\end{array}\right) S_{1}(l, m)\left(( \sum _ { \nu = 1 } ^ { r } a _ { v } ) \left\langle\frac{(1+t)^{a_{\nu}} \ln (1+t)}{(1+t)^{a_{\nu}}-1} \prod_{i=1}^{r}\left(\frac{(1+t)^{a_{i}}-1}{(1+t)^{a_{i}} \ln (1+t)}\right)\right.\right. \\
& \times \prod_{j=1}^{s}\left(\frac{(1+t)^{\lambda_{j}}}{1+(1+t)^{\lambda_{j}}}\right)^{\mu_{j}}(1+t)^{-1}\left|\frac{t}{\ln (1+t)} x^{n-l}\right\rangle \\
& \left.-r\left\langle\prod_{i=1}^{r}\left(\frac{(1+t)^{a_{i}}-1}{(1+t)^{a_{i}} \ln (1+t)}\right) \prod_{j=1}^{s}\left(\frac{(1+t)^{\lambda_{j}}}{1+(1+t)^{\lambda_{j}}}\right)^{\mu_{j}}(1+t)^{-1} \mid \frac{t}{\ln (1+t)} x^{n-l}\right\rangle\right) \\
& =-m !\left(\sum_{\nu=1}^{r} a_{v}\right) \sum_{l=m}^{n-1}\left(\begin{array}{c}
n-1 \\
l
\end{array}\right) S_{1}(l, m) \widehat{\mathrm{NS}}_{n-l-1}(-1) \\
& +\frac{m !}{n} \sum_{l=m}^{n}\left(\begin{array}{l}
n \\
l
\end{array}\right) S_{1}(l, m) \sum_{k=0}^{n-l}\left(\begin{array}{c}
n-l \\
k
\end{array}\right) c_{k} \\
& \times\left(\left(\sum_{\nu=1}^{r} a_{\nu}\right) \widehat{\mathrm{NS}}_{n-l-k}\left(-1 \mid a_{1}, \ldots, a_{i-1}, a_{i+1}, \ldots, a_{r} ; \lambda ; \mu\right)-r \widehat{\mathrm{NS}}_{n-l-k}(-1 \mid a ; \lambda ; \mu)\right) \\
& =-m !\left(\sum_{\nu=1}^{r} a_{\nu}\right) \sum_{l=0}^{n-1-m}\left(\begin{array}{c}
n-1 \\
l
\end{array}\right) S_{1}(n-l-1, m) \widehat{\mathrm{NS}}_{l}(-1) \\
& +\frac{m !}{n} \sum_{k=0}^{n-m} \sum_{l=k}^{n-m}\left(\begin{array}{l}
n \\
l
\end{array}\right)\left(\begin{array}{l}
l \\
k
\end{array}\right) S_{1}(n-l, m) c_{l-k} \\
& \times\left(\left(\sum_{\nu=1}^{r} a_{\nu}\right) \widehat{\mathrm{NS}}_{k}\left(-1 \mid a_{1}, \ldots, a_{i-1}, a_{i+1}, \ldots, a_{r} ; \lambda ; \mu\right)-r \widehat{\mathrm{NS}}_{k}(-1 \mid a ; \lambda ; \mu)\right) .
\end{aligned}
$$

The second term of (28) is equal to

$$
\begin{aligned}
& \sum_{\nu=1}^{s} \lambda_{\nu} \mu_{\nu}\left\langle\prod_{i=1}^{r}\left(\frac{(1+t)^{a_{i}}-1}{(1+t)^{a_{i}} \ln (1+t)}\right)\left(\frac{(1+t)^{\lambda_{\nu}}}{1+(1+t)^{\lambda_{\nu}}}\right)\right. \\
& \times \prod_{j=1}^{s}\left(\frac{(1+t)^{\lambda_{j}}}{1+(1+t)^{\lambda_{j}}}\right)^{\mu_{j}}(1+t)^{-\lambda_{\nu}-1}\left|(\ln (1+t))^{m} x^{n-1}\right\rangle \\
& =\sum_{\nu=1}^{s} \lambda_{\nu} \mu_{\nu}\left\langle\prod_{i=1}^{r}\left(\frac{(1+t)^{a_{i}}-1}{(1+t)^{a_{i}} \ln (1+t)}\right)\left(\frac{(1+t)^{\lambda_{\nu}}}{1+(1+t)^{\lambda_{\nu}}}\right)\right. \\
& \times \prod_{j=1}^{s}\left(\frac{(1+t)^{\lambda_{j}}}{1+(1+t)^{\lambda_{j}}}\right)^{\mu_{j}}(1+t)^{-\lambda_{v}-1}\left|m ! \sum_{l=m}^{\infty} S_{1}(l, m) \frac{t^{l}}{l !} x^{n-1}\right\rangle \\
& =m ! \sum_{\nu=1}^{s} \lambda_{\nu} \mu_{\nu} \sum_{l=m}^{n-1}\left(\begin{array}{c}
n-1 \\
l
\end{array}\right) S_{1}(l, m)\left\langle\prod_{i=1}^{r}\left(\frac{(1+t)^{a_{i}}-1}{(1+t)^{a_{i}} \ln (1+t)}\right)\left(\frac{(1+t)^{\lambda_{\nu}}}{1+(1+t)^{\lambda_{\nu}}}\right)\right. \\
& \times \prod_{j=1}^{s}\left(\frac{(1+t)^{\lambda_{j}}}{1+(1+t)^{\lambda_{j}}}\right)^{\mu_{j}}(1+t)^{-\lambda_{v}-1}\left|x^{n-l-1}\right\rangle \\
& =m ! \sum_{\nu=1}^{s} \lambda_{\nu} \mu_{v} \sum_{l=m}^{n-1}\left(\begin{array}{c}
n-1 \\
l
\end{array}\right) S_{1}(l, m) \widehat{\mathrm{NS}}_{n-l-1}\left(-\lambda_{v}-1 \mid a ; \lambda ; \mu+e_{\nu}\right) \\
& =m ! \sum_{\nu=1}^{s} \lambda_{\nu} \mu_{\nu} \sum_{l=0}^{n-m-1}\left(\begin{array}{c}
n-1 \\
l
\end{array}\right) S_{1}(n-l-1, m) \widehat{\mathrm{NS}}_{l}\left(-\lambda_{v}-1 \mid a ; \lambda ; \mu+e_{v}\right) .
\end{aligned}
$$


Therefore, we get, for $n-1 \geq m \geq 1$,

$$
\begin{aligned}
& m ! \sum_{l=0}^{n-m}\left(\begin{array}{l}
n \\
l
\end{array}\right) S_{1}(n-l, m) \widehat{\mathrm{NS}}_{l}(a ; \lambda ; \mu) \\
& =-m !\left(\sum_{i=1}^{r} a_{i}\right) \sum_{l=0}^{n-m-1}\left(\begin{array}{c}
n-1 \\
l
\end{array}\right) S_{1}(n-l-1, m) \widehat{\mathrm{NS}}_{l}(-1 \mid a ; \lambda ; \mu) \\
& +\frac{m !}{n} \sum_{k=0}^{n-m} \sum_{l=k}^{n-m}\left(\begin{array}{c}
n \\
l
\end{array}\right)\left(\begin{array}{l}
l \\
k
\end{array}\right) S_{1}(n-l, m) c_{l-k} \\
& \quad \times\left(\sum_{i=1}^{r} a_{i} \widehat{\mathrm{NS}}_{k}\left(-1 \mid a_{1}, \ldots, a_{i-1}, a_{i+1}, \ldots, a_{r} ; \lambda ; \mu\right)-r \widehat{\mathrm{NS}}_{k}(-1 \mid a ; \lambda ; \mu)\right) \\
& \quad+m ! \sum_{j=1}^{s} \lambda_{j} \mu_{j} \sum_{l=0}^{n-m-1}\left(\begin{array}{c}
n-1 \\
l
\end{array}\right) S_{1}(n-l-1, m) \widehat{\mathrm{NS}}_{l}\left(-\lambda,-1 \mid a ; \lambda ; \mu+e_{j}\right) \\
& \quad+m ! \sum_{l=0}^{n-m}\left(\begin{array}{c}
n-1 \\
l
\end{array}\right) S_{1}(n-l-1, m-1) \widehat{\mathrm{NS}}_{l}(-1 \mid a ; \lambda ; \mu) .
\end{aligned}
$$

Dividing both sides by $m$ !, we obtain, for $n-1 \geq m \geq 1$,

$$
\begin{aligned}
\sum_{l=0}^{n-m}\left(\begin{array}{l}
n \\
l
\end{array}\right) S_{1}(n-l, m) \widehat{\mathrm{NS}}_{l}(a ; \lambda ; \mu) \\
=-\left(\sum_{i=1}^{r} a_{i}\right) \sum_{l=0}^{n-m-1}\left(\begin{array}{c}
n-1 \\
l
\end{array}\right) S_{1}(n-l-1, m) \widehat{\mathrm{NS}}_{l}(-1 \mid a ; \lambda ; \mu) \\
+\frac{1}{n} \sum_{k=0}^{n-m} \sum_{l=k}^{n-m}\left(\begin{array}{l}
n \\
l
\end{array}\right)\left(\begin{array}{l}
l \\
k
\end{array}\right) S_{1}(n-l, m) c_{l-k} \\
\quad \times\left(\sum_{i=1}^{r} a_{i} \widehat{\mathrm{NS}}_{k}\left(-1 \mid a_{1}, \ldots, a_{i-1}, a_{i+1}, \ldots, a_{r} ; \lambda ; \mu\right)-r \widehat{\mathrm{NS}}_{k}(-1 \mid a ; \lambda ; \mu)\right) \\
+\sum_{j=1}^{s} \lambda_{j} \mu_{j} \sum_{l=0}^{n-m-1}\left(\begin{array}{c}
n-1 \\
l
\end{array}\right) S_{1}(n-l-1, m) \widehat{\mathrm{NS}}_{l}\left(-\lambda j-1 \mid a ; \lambda ; \mu+e_{j}\right) \\
+\sum_{l=0}^{n-m}\left(\begin{array}{c}
n-1 \\
l
\end{array}\right) S_{1}(n-l-1, m-1) \widehat{\mathrm{NS}}_{l}(-1 \mid a ; \lambda ; \mu) .
\end{aligned}
$$

Thus, we get (27).

\subsection{A relation with the falling factorials}

\section{Theorem 8}

$$
\begin{aligned}
& \widehat{\mathrm{NS}}_{n}\left(x \mid a_{1}, \ldots, a_{r} ; \lambda_{1}, \ldots, \lambda_{s} ; \mu_{1}, \ldots, \mu_{s}\right) \\
& \quad=\sum_{m=0}^{n}\left(\begin{array}{l}
n \\
m
\end{array}\right) \widehat{\mathrm{NS}}_{n-m}\left(a_{1}, \ldots, a_{r} ; \lambda_{1}, \ldots, \lambda_{s} ; \mu_{1}, \ldots, \mu_{s}\right)(x)_{m} .
\end{aligned}
$$


Proof For (12) and (20), assume that

$$
\widehat{\mathrm{NS}}_{n}\left(x \mid a_{1}, \ldots, a_{r} ; \lambda_{1}, \ldots, \lambda_{s} ; \mu_{1}, \ldots, \mu_{s}\right)=\sum_{m=0}^{n} C_{n, m}(x)_{m}
$$

By (11), we have

$$
\begin{aligned}
C_{n, m} & =\frac{1}{m !}\left\langle\frac{1}{\prod_{i=1}^{r}\left(\frac{\ln (1+t) e^{a_{i} \ln (1+t)}}{e^{a_{i} \ln (1+t)}-1}\right) \prod_{j=1}^{s}\left(\frac{1+e^{\lambda_{j} \ln (1+t)}}{e^{\lambda_{j} \ln (1+t)}}\right)^{\mu_{j}}} t^{m} \mid x^{n}\right\rangle \\
& =\frac{1}{m !}\left\langle\prod_{i=1}^{r}\left(\frac{(1+t)^{a_{i}}-1}{(1+t)^{a_{i}} \ln (1+t)}\right) \prod_{j=1}^{s}\left(\frac{(1+t)^{\lambda_{j}}}{1+(1+t)^{\lambda_{j}}}\right)^{\mu_{j}} \mid t^{m} x^{n}\right\rangle \\
& =\left(\begin{array}{c}
n \\
m
\end{array}\right)\left\langle\prod_{i=1}^{r}\left(\frac{(1+t)^{a_{i}}-1}{(1+t)^{a_{i}} \ln (1+t)}\right) \prod_{j=1}^{s}\left(\frac{(1+t)^{\lambda_{j}}}{1+(1+t)^{\lambda_{j}}}\right)^{\mu_{j}} \mid x^{n-m}\right\rangle \\
& =\left(\begin{array}{c}
n \\
m
\end{array}\right) \widehat{\mathrm{NS}}_{n-m .}
\end{aligned}
$$

Thus, we get the identity (29).

\subsection{A relation with higher-order Frobenius-Euler polynomials}

For $\alpha \in \mathbb{C}$ with $\alpha \neq 1$, the Frobenius-Euler polynomials of order $r, H_{n}^{(r)}(x \mid \alpha)$ are defined by the generating function

$$
\left(\frac{1-\alpha}{e^{t}-\alpha}\right)^{r} e^{x t}=\sum_{n=0}^{\infty} H_{n}^{(r)}(x \mid \alpha) \frac{t^{n}}{n !}
$$

(see e.g. [5]).

\section{Theorem 9}

$$
\begin{aligned}
& \widehat{\mathrm{NS}}_{n}\left(x \mid a_{1}, \ldots, a_{r} ; \lambda_{1}, \ldots, \lambda_{s} ; \mu_{1}, \ldots, \mu_{s}\right) \\
& \quad=\sum_{m=0}^{n}\left(\sum_{j=0}^{n-m} \sum_{l=0}^{n-m-j}\left(\begin{array}{c}
\sigma \\
j
\end{array}\right)\left(\begin{array}{c}
n-j \\
l
\end{array}\right)(n)_{j}(1-\alpha)^{-j} S_{1}(n-j-l, m) \widehat{\mathrm{NS}}_{l}\right) H_{m}^{(\sigma)}(x \mid \alpha) .
\end{aligned}
$$

Proof For (12) and

$$
H_{n}^{(\sigma)}(x \mid \alpha) \sim\left(\left(\frac{e^{t}-\alpha}{1-\alpha}\right)^{\sigma}, t\right)
$$

assume that $\widehat{\mathrm{NS}}_{n}\left(x \mid a_{1}, \ldots, a_{r} ; \lambda_{1}, \ldots, \lambda_{s} ; \mu_{1}, \ldots, \mu_{s}\right)=\sum_{m=0}^{n} C_{n, m} H_{m}^{(\sigma)}(x \mid \alpha)$. By (11), similarly to the proof of (27), we have

$$
\begin{aligned}
C_{n, m} & =\frac{1}{m !}\left\langle\frac{\left(\frac{e^{\ln (1+t)}-\alpha}{1-\alpha}\right)^{\sigma}}{\prod_{i=1}^{r}\left(\frac{\ln (1+t) e^{a_{i} \ln (1+t)}}{e^{a_{i} \ln (1+t)}-1}\right) \prod_{j=1}^{s}\left(\frac{1+e^{\lambda_{j} \ln (1+t)}}{e^{\lambda_{j} \ln (1+t)}}\right)^{\mu_{j}}}(\ln (1+t))^{m} \mid x^{n}\right\rangle \\
& =\frac{1}{m !(1-\alpha)^{\sigma}}
\end{aligned}
$$




$$
\begin{aligned}
& \times\left\langle\prod_{i=1}^{r}\left(\frac{(1+t)^{a_{i}}-1}{(1+t)^{a_{i}} \ln (1+t)}\right) \prod_{j=1}^{s}\left(\frac{(1+t)^{\lambda_{j}}}{1+(1+t)^{\lambda_{j}}}\right)^{\mu_{j}}(\ln (1+t))^{m}(1-\alpha+t)^{\sigma} \mid x^{n}\right\rangle \\
= & \frac{1}{m !(1-\alpha)^{\sigma}}\left\langle\prod_{i=1}^{r}\left(\frac{(1+t)^{a_{i}}-1}{(1+t)^{a_{i}} \ln (1+t)}\right) \prod_{j=1}^{s}\left(\frac{(1+t)^{\lambda_{j}}}{1+(1+t)^{\lambda_{j}}}\right)^{\mu_{j}}\right. \\
& \times(\ln (1+t))^{m}\left|\sum_{v=0}^{m \min \{\sigma, n\}}\left(\begin{array}{l}
\sigma \\
v
\end{array}\right)(1-\alpha)^{\sigma-v} t^{\nu} x^{n}\right\rangle \\
= & \frac{1}{m !(1-\alpha)^{\sigma}} \sum_{v=0}^{n-m}\left(\begin{array}{c}
\sigma \\
v
\end{array}\right)(1-\alpha)^{\sigma-v}(n)_{v} \\
& \times\left\langle\prod_{i=1}^{r}\left(\frac{(1+t)^{a_{i}}-1}{(1+t)^{a_{i}} \ln (1+t)}\right) \prod_{j=1}^{s}\left(\frac{(1+t)^{\lambda_{j}}}{1+(1+t)^{\lambda_{j}}}\right)^{\mu_{j}}(\ln (1+t))^{m} \mid x^{n-v}\right\rangle \\
= & \frac{1}{m !(1-\alpha)^{\sigma}} \sum_{v=0}^{n-m}\left(\begin{array}{c}
\sigma \\
v
\end{array}\right)(1-\alpha)^{\sigma-v}(n)_{v} \sum_{l=0}^{n-m-v} m !\left(\begin{array}{c}
n-v \\
l
\end{array}\right) S_{1}(n-v-l, m) \widehat{\mathrm{NS}} l \\
= & \sum_{v=0}^{n-m} \sum_{l=0}^{n-m-v}\left(\begin{array}{c}
\sigma \\
v
\end{array}\right)\left(\begin{array}{c}
n-v \\
l
\end{array}\right)(n)_{v}(1-\alpha)^{-v} S_{1}(n-v-l, m) \widehat{\mathrm{NS}}_{l} .
\end{aligned}
$$

Thus, we get the identity (30).

\subsection{A relation with higher-order Bernoulli polynomials}

Bernoulli polynomials $\mathfrak{B}_{n}^{(r)}(x)$ of order $r$ are defined by

$$
\left(\frac{t}{e^{t}-1}\right)^{r} e^{x t}=\sum_{n=0}^{\infty} \frac{\mathfrak{B}_{n}^{(r)}(x)}{n !} t^{n}
$$

(see e.g. [1, Section 2.2]). In addition, the Cauchy numbers of the first kind $\mathfrak{C}_{n}^{(r)}$ of order $r$ are defined by

$$
\left(\frac{t}{\ln (1+t)}\right)^{r}=\sum_{n=0}^{\infty} \frac{\mathfrak{C}_{n}^{(r)}}{n !} t^{n}
$$

(see e.g. [6, equation (2.1)], [7, equation (6)]).

\section{Theorem 10}

$$
\begin{aligned}
& \widehat{\mathrm{NS}}_{n}\left(x \mid a_{1}, \ldots, a_{r} ; \lambda_{1}, \ldots, \lambda_{s} ; \mu_{1}, \ldots, \mu_{s}\right) \\
& \quad=\sum_{m=0}^{n}\left(\sum_{i=0}^{n-m} \sum_{l=0}^{n-m-i}\left(\begin{array}{c}
n \\
i
\end{array}\right)\left(\begin{array}{c}
n-i \\
l
\end{array}\right) \mathfrak{C}_{i}^{(\sigma)} S_{1}(n-i-l, m) \widehat{\mathrm{NS}}_{l}\right) \mathfrak{B}_{m}^{(\sigma)}(x) .
\end{aligned}
$$

Proof For (12) and

$$
\mathfrak{B}_{n}^{(\sigma)}(x) \sim\left(\left(\frac{e^{t}-1}{t}\right)^{\sigma}, t\right)
$$


assume that $\widehat{\mathrm{NS}}_{n}\left(x \mid a_{1}, \ldots, a_{r} ; \lambda_{1}, \ldots, \lambda_{s} ; \mu_{1}, \ldots, \mu_{s}\right)=\sum_{m=0}^{n} C_{n, m} \mathfrak{B}_{m}^{(s)}(x)$. By (11), similarly to the proof of (27), we have

$$
\begin{aligned}
& C_{n, m}=\frac{1}{m !}\left\langle\frac{\left(\frac{e^{\ln (1+t)}-1}{\ln (1+t)}\right)^{\sigma}}{\prod_{i=1}^{r}\left(\frac{\ln (1+t) e^{a_{i} \ln (1+t)}}{e^{a_{i} \ln (1+t)}-1}\right) \prod_{j=1}^{s}\left(\frac{1+e^{\lambda_{j} \ln (1+t)}}{e^{\lambda_{j} \ln (1+t)}}\right)^{\mu_{j}}}(\ln (1+t))^{m} \mid x^{n}\right\rangle \\
& =\frac{1}{m !}\left\langle\prod_{i=1}^{r}\left(\frac{(1+t)^{a_{i}}-1}{(1+t)^{a_{i}} \ln (1+t)}\right) \prod_{j=1}^{s}\left(\frac{(1+t)^{\lambda_{j}}}{1+(1+t)^{\lambda_{j}}}\right)^{\mu_{j}}(\ln (1+t))^{m} \mid\left(\frac{t}{\ln (1+t)}\right)^{\sigma} x^{n}\right\rangle \\
& =\frac{1}{m !}\left\langle\prod_{i=1}^{r}\left(\frac{(1+t)^{a_{i}}-1}{(1+t)^{a_{i}} \ln (1+t)}\right) \prod_{j=1}^{s}\left(\frac{(1+t)^{\lambda_{j}}}{1+(1+t)^{\lambda_{j}}}\right)^{\mu_{j}}(\ln (1+t))^{m} \mid \sum_{i=0}^{\infty} \mathfrak{C}_{i}^{(\sigma)} \frac{t^{i}}{i !} x^{n}\right\rangle \\
& =\frac{1}{m !} \sum_{i=0}^{n-m} \mathfrak{C}_{i}^{(\sigma)}\left(\begin{array}{l}
n \\
i
\end{array}\right)\left\langle\prod_{i=1}^{r}\left(\frac{(1+t)^{a_{i}}-1}{(1+t)^{a_{i}} \ln (1+t)}\right) \prod_{j=1}^{s}\left(\frac{(1+t)^{\lambda_{j}}}{1+(1+t)^{\lambda_{j}}}\right)^{\mu_{j}}(\ln (1+t))^{m} \mid x^{n-i}\right\rangle \\
& =\frac{1}{m !} \sum_{i=0}^{n-m} \mathfrak{C}_{i}^{(\sigma)}\left(\begin{array}{c}
n \\
i
\end{array}\right) \sum_{l=0}^{n-m-i} m !\left(\begin{array}{c}
n-i \\
l
\end{array}\right) S_{1}(n-i-l, m) \widehat{\mathrm{NS}}_{l} \\
& =\sum_{i=0}^{n-m} \sum_{l=0}^{n-m-i}\left(\begin{array}{c}
n \\
i
\end{array}\right)\left(\begin{array}{c}
n-i \\
l
\end{array}\right) \mathfrak{C}_{i}^{(\sigma)} S_{1}(n-i-l, m) \widehat{\mathrm{NS}}_{l} .
\end{aligned}
$$

Thus, we get the identity (32).

\section{Competing interests}

The authors declare that they have no competing interests.

\section{Authors' contributions}

All authors contributed equally to this work. All authors read and approved the final manuscript.

\section{Author details}

'Department of Mathematics, Sogang University, Seoul, 121-741, Republic of Korea. ${ }^{2}$ Department of Mathematics, Kwangwoon University, Seoul, 139-701, Republic of Korea. ${ }^{3}$ Graduate School of Science and Technology, Hirosaki University, Hirosaki, 036-8561, Japan.

\section{Acknowledgements}

The work reported in this paper was conducted during the sabbatical year of Kwangwoon University in 2014.

Received: 23 July 2014 Accepted: 10 September 2014 Published: 29 Sep 2014

\section{References}

1. Roman, S: The Umbral Calculus. Dover, New York (2005)

2. Kim, DS, Kim, T: Poly-Cauchy and Peters mixed-type polynomials. Adv. Differ. Equ. 2014, 4 (2014)

3. Comtet, L: Advanced Combinatorics. Reidel, Dordrecht (1974)

4. Kim, DS, Kim, T: Higher-order Cauchy of the first kind and poly-Cauchy of the first kind mixed type polynomials. Adv. Stud. Contemp. Math. 23(4), 621-636 (2013)

5. Kim, DS, Kim, T: Some identities of Frobenius-Euler polynomials arising from umbral calculus. Adv. Differ. Equ. 2012, 196 (2012)

6. Carlitz, L: A note on Bernoulli and Euler polynomials of the second kind. Scr. Math. 25, 323-330 (1961)

7. Liang, H, Wuyungaowa: Identities involving generalized harmonic numbers and other special combinatorial sequences. J. Integer Seq. 15, Article 12.9.6 (2012)

10.1186/1029-242X-2014-376

Cite this article as: Kim et al.: Barnes-type Narumi of the second kind and Barnes-type Peters of the second kind hybrid polynomials. Journal of Inequalities and Applications 2014, 2014:376 